\title{
Relating Innermost, Weak, Uniform and Modular Termination of Term Rewriting Systems
}

\author{
Bernhard Gramlich* \\ Fachbereich Informatik, Universität Kaiserslautern \\ Erwin-Schroedinger-Str., 67663 Kaiserslautern, Germany \\ gramlich@informatik.uni-kl.de
}

June 10, 1993

\begin{abstract}
We investigate restricted termination and confluence properties of term rewriting systems, in particular weak termination and innermost termination, and their interrelation. New criteria are provided which are sufficient for the equivalence of innermost / weak termination and uniform termination of term rewriting systems. These criteria provide interesting possibilities to infer completeness, i.e. termination plus confluence, from restricted termination and confluence properties.

Using these basic results we are also able to prove some new results about modular termination of rewriting. In particular, we show that termination is modular for some classes of innermost terminating and locally confluent term rewriting systems, namely for non-overlapping and even for overlay systems. As an easy consequence this latter result also entails a simplified proof of the fact that completeness is a decomposable property of so-called constructor systems. Furthermore we show how to obtain similar results for even more general cases of (non-disjoint) combined systems with shared constructors and of certain hierarchical combinations of systems with constructors. Interestingly, these modularity results are obtained by means of a proof technique which itself constitutes a modular approach.
\end{abstract}

Key Words: Term rewriting systems, confluence, termination, weak termination, innermost termination, modularity, disjoint union, combined systems with shared constructors, constructor systems, hierarchical combinations.

\footnotetext{
${ }^{*}$ This research was supported by the 'Deutsche Forschungsgemeinschaft, SFB 314 (D4-Projekt)'.
} 


\section{Introduction}

Term rewriting systems play an important role in various areas, e.g. in abstract data type specifications, for automated theorem proving and as a basic computation model for functional programming languages. In theory and practice, one of the most important properties of term rewriting systems is the strong normalization or (finite or uniform) termination property which is undecidable in general. For ensuring this property which is undecidable in general (see [HL78]), many sufficient criteria, techniques and methods have been developed (see [Der87] for a survey). Most practically applicable approaches are based on reduction orderings, i.e. well-founded term orderings which are stable w.r.t. substitutions and monotonic w.r.t. the term structure.

On the other hand, in many rewriting based computation models, e.g. in functional programming languages, the indeterminism of general rewriting is often restricted by imposing some fixed rewriting strategy. For instance, a frequent restriction is innermost reduction, i.e. to require that every reduction step takes place at an innermost position of the term to be reduced. Innermost reduction corresponds closely to the functional evaluation mechanism employed in functional programming languages like LISP or ML. Of course, it may be the case that correspondingly restricted computations, i.e. innermost reduction sequences, always terminate but arbitrary computations (reduction sequences) do not necessarily terminate. A very simple example illustrating this gap is the following:

Example 1.1 Let $R=\{f(a) \rightarrow f(a), a \rightarrow b\}$. Then we have e.g. the infinite reduction sequence $f(a) \rightarrow f(a) \rightarrow f(a) \rightarrow \ldots$, which uses only non-innermost reduction steps. But of course, every innermost derivation in $R$ (e.g. $f(a) \rightarrow f(b))$ is terminating.

Other kinds of restrictions imposed on rewriting steps might also be conceivable according to the intended purpose, e.g. leftmost outermost, top-down, bottom-up or other context-dependent strategies. Unfortunately, very little is known about termination of rewriting under such restrictions and its relation to (uniform) termination. In fact, there is one major exception, namely concerning the important and thoroughly investigated class of so-called orthogonal TRSs, i.e. TRSs which are left-linear and non-overlapping (see [Klo92] for a survey of basic ideas, concepts and results about the theory of orthogonal TRSs). It is well-known that any orthogonal TRSs is confluent notwithstanding the fact that it may be non-terminating. For arbitrary TRSs one can conclude in general nothing about confluence ${ }^{1}$ or the existence of (unique) normal forms if termination is not guaranteed since these properties are undecidable in the general case.

In the following we shall study in particular under what conditions innermost termination implies (uniform) termination of rewriting. More generally, we shall investigate and develop some extensions and generalizations of known results about orthogonal

\footnotetext{
${ }^{1}$ Of course, this phenomenon is due to the fact that in general confluence and local confluence need not coincide. Hence, the critical pair test for ensuring local confluence is not sufficient for confluence.
} 
TRSs. This is done by weakening both the no-overlap and the left-linearity requirement but still guaranteeing local confluence. Moreover, in a second part of the paper we shall apply these abstract results in a modular fashion in order to obtain new sufficient criteria for modular termination of TRSs and corresponding invariance properties of certain classes of non-disjoint combinations of TRSs. Before going into details let us give a summary of our main results: ${ }^{2}$

- If a TRS $\mathcal{R}$ is non-overlapping then weak innermost termination of $\mathcal{R}$ is equivalent to innermost termination of $\mathcal{R}$ (see Lemma 3.5).

- If a TRS $\mathcal{R}$ is non-overlapping and innermost terminating then it is (uniformly) terminating (and confluent, hence complete) (see Lemma 3.7 and Theorem 3.10).

- If a TRS $\mathcal{R}$ is non-overlapping, weakly terminating and non-erasing then it is terminating (and hence confluent and complete) (see Theorem 3.13).

- If a TRS $\mathcal{R}$ is an innermost terminating overlay system with joinable critical pairs then it is (uniformly) terminating (and hence confluent and complete) (see Theorem 3.20).

- If a TRS $\mathcal{R}$ is an innermost terminating constructor system with joinable critical pairs then it is (uniformly) terminating (and hence confluent and complete) (see corollary 4.6).

- Innermost termination is a modular property of TRSs (see Lemma 4.2).

- Termination (and hence completeness) is modular for locally confluent overlay systems (see Theorem 4.4).

- The union of two constructor systems with disjoint sets of defined symbols is complete if and only if both systems are complete (see Theorem 4.8). ${ }^{3}$

- A combined system with shared constructors is a complete overlay system if and only if its component systems are complete overlay systems (see Theorem 4.11).

- A weakly separated hierarchical combination of TRSs is a complete overlay system if and only if its component systems are complete overlay systems (see Theorem 4.28$).^{4}$

The rest of the paper which is an extended version of [Gra92b] is structured as follows. Firstly, we introduce the basic definitions and notions needed later on. In section 3 we study the innermost, weak and uniform termination properties of TRSs which are non-overlapping but not necessarily left-linear. More generally we also investigate the termination behaviour of certain restricted classes of (possibly overlapping) locally confluent TRSs.

\footnotetext{
${ }^{2}$ The definitions involved here are presented below.

${ }^{3}$ This result has been obtained in [MT91] by means of a more direct and rather intricate proof.

${ }^{4}$ Cf. [Der92], [Kri92] for slightly weaker, related results.
} 
Then, in section 4, we consider modular properties of TRSs, applying previously developed ideas and results. And finally, related work as well as some open problems are discussed.

\section{Preliminaries}

We briefly recall the basic terminology needed for dealing with TRSs (see e.g. [Klo92], [DJ90]). Let $\mathcal{V}$ be a countably infinite set of variables and $\mathcal{F}$ be a set of function symbols with $\mathcal{V} \cap \mathcal{F}=\emptyset$. Associated to every $f \in \mathcal{F}$ is a natural number denoting its arity. Function symbols of arity 0 are called constants. The set $\mathcal{T}(\mathcal{F}, \mathcal{V})$ of terms over $\mathcal{F}$ and $\mathcal{V}$ is the smallest set with $(1) \mathcal{V} \subseteq \mathcal{T}(\mathcal{F}, \mathcal{V})$ and (2) if $f \in \mathcal{F}$ has arity $\mathrm{n}$ and $t_{1}, \ldots, t_{n} \in \mathcal{T}(\mathcal{F}, \mathcal{V})$ then $f\left(t_{1}, \ldots, t_{n}\right) \in \mathcal{T}(\mathcal{F}, \mathcal{V})$. If some function symbols are allowed to be varyadic then the definition of $\mathcal{T}(\mathcal{F}, \mathcal{V})$ is generalized in an obvious way. The set of all ground terms (over $\mathcal{F}$ ), i.e. terms with no variables, is denoted by $\mathcal{T}(\mathcal{F}$ ). In the following we shall always assume that $\mathcal{T}(\mathcal{F})$ is non-empty, i.e. there is at least one constant in $\mathcal{F}$. The set of variables (function symbols) occurring in a term $\mathrm{t}$ is denoted by $V(t)(F(t))$. The top symbol of a term $t$ is denoted by $\operatorname{root}(t)$.

A context $C[, \ldots$,$] is a term with 'holes', i.e. a term in \mathcal{T}(\mathcal{F} \uplus\{\square\}, \mathcal{V})^{5}$ where $\square$ is a new special constant symbol. If $C[, \ldots$,$] is a context with \mathrm{n}$ occurrences of $\square$ and $t_{1}, \ldots, t_{n}$ are terms then $C\left[t_{1}, \ldots, t_{n}\right]$ is the term obtained from $C[, \ldots$,$] by replacing$ from left to right the occurrences of $\square$ by $t_{1}, \ldots, t_{n}$. A context containing precisely one occurrence of $\square$ is denoted by $C[]$. For the set $\mathcal{T}(\mathcal{F} \uplus\{\square\}, \mathcal{V})$ we also write $\mathcal{C O N}(\mathcal{F}, \mathcal{V})$. A non-empty context is a term from $\mathcal{C O N}(\mathcal{F}, \mathcal{V}) \backslash \mathcal{T}(\mathcal{F}, \mathcal{V})$ which is different from $\square$. A term $s$ is a subterm of a term $t$ if there exists a context $C[]$ with $t=C[s]$. If in addition $C[] \neq \square$ then $s$ is a proper subterm of t. A substitution $\sigma$ is a mapping from $\mathcal{V}$ to $\mathcal{T}(\mathcal{F}, \mathcal{V})$ such that its domain $\operatorname{dom}(\sigma)=\{x \in \mathcal{V} \mid \sigma x \neq x\}$ is finite. Its homomorphic extension to a mapping from $\mathcal{T}(\mathcal{F}, \mathcal{V})$ to $\mathcal{T}(\mathcal{F}, \mathcal{V})$ is also denoted by $\sigma$.

A term rewriting system $(T R S)$ is a pair $(\mathcal{R}, \mathcal{F})$ consisting of a signature $\mathcal{F}^{6}$ and a set $\mathcal{R} \subseteq \mathcal{T}(\mathcal{F}, \mathcal{V}) \times \mathcal{T}(\mathcal{F}, \mathcal{V})$ of (rewrite) rules $(l, r)$ denoted by $l \rightarrow r$ with $l \notin \mathcal{V}$ and $V(r) \subseteq V(l) .{ }^{7}$ Instead of $(\mathcal{R}, \mathcal{F})$ we also write $\mathcal{R}^{\mathcal{F}}$ or simply $\mathcal{R}$ when $\mathcal{F}$ is clear from the context or irrelevant.

Given a TRS $\mathcal{R}^{\mathcal{F}}$ the rewrite relation $\rightarrow_{\mathcal{R}^{\mathcal{F}}}$ for terms $s, t \in \mathcal{T}(\mathcal{F}, \mathcal{V})$ is defined as follows: $s \rightarrow \mathcal{R}^{\mathcal{F}} t$ if there exists a rule $l \rightarrow r \in \mathcal{R}$, a substitution $\sigma$ and a context $C[]$ such that $s=C[\sigma l]$ and $t=C[\sigma r]$. We also write $\rightarrow \mathcal{R}$ or simply $\rightarrow$ when $\mathcal{F}$ or $\mathcal{R}^{\mathcal{F}}$ is clear from the context, respectively. The symmetric, transitive, transitive-reflexive and symmetric-transitive-reflexive closures of $\rightarrow$ are denoted by $\leftrightarrow, \rightarrow^{+}, \rightarrow^{*}$ and $\stackrel{*}{\leftrightarrow}$, respectively. By $s \rightarrow^{m} t$ we mean that $s$ is reduced to $t$ in $m$ steps. Accordingly $s \rightarrow{ }^{\leq n} t$ means $s \rightarrow^{m} t$ for some $m \leq n$. Two terms $s, t$ are joinable in $\mathcal{R}$, denoted by

\footnotetext{
${ }^{5}$ The symbol $\uplus$ is to denote disjoint union.

${ }^{6}$ Here and subsequently we always tacitly assume that some (countably infinite) set $\mathcal{V}$ of variables with $\mathcal{V} \cap \mathcal{F}=\emptyset$ is given.

${ }^{7}$ This restriction of excluding variable left-hand sides and right-hand side extra-variables is not a severe one. In particular, concerning termination of rewriting it only excludes trivial cases.
} 
$s \downarrow_{\mathcal{R}} t$, if there exists a term $u$ with $s \rightarrow_{\mathcal{R}}^{*} u{ }_{\mathcal{R}}^{*} \leftarrow t$. A term $s$ is irreducible or in normal form if there is no term $t$ with $s \rightarrow t$. A term $t$ is said to be a normal form of a term $s$ if $s \rightarrow^{*} t$ and $t$ is irreducible. A TRS $\mathcal{R}$ is terminating or strongly normalizing (SN) if $\rightarrow$ is noetherian, i.e. if there is no infinite reduction sequence $s_{1} \rightarrow s_{2} \rightarrow s_{3} \rightarrow \cdots$. It is said to be weakly terminating or weakly normalizing $(W N)$ if for every term there exists a normal form. Positions or occurrences of subterms of a term consist of sequences of natural numbers and are compared by the usual lexicographic ordering (which we shall ambiguously denote by $\leq$ ). The set of all positions of a term $s$ is denoted by $O(s)$. The topmost position of a term is denoted by $\lambda$, the 'empty' string. Two uncomparable positions $p$ and $q$ are said to be parallel or disjoint which is denoted by $p \mid q$. If $p \leq q$ we say that $p$ is above $q$ or $q$ is below $p$. If $s \rightarrow t$, then, in order to make explicit the position $p$ of the reduced subterm and the applied rule $l \rightarrow r$, we shall sometimes use the notation $s \rightarrow_{p, l \rightarrow r} t$ or $s \rightarrow_{p} t$. A step of the form $s \rightarrow_{\lambda} t$ is said to be a root reduction (step). If $s \rightarrow_{p} t$ then the reduced subterm $s / p$ of $s$ is said to be a redex. ${ }^{8}$ A reduction step $s \rightarrow t$ by applying some rule of $\mathcal{R}$ at position $p$ in $s$ is innermost if every proper subterm of $s / p$ is irreducible. In that case we also write $s \underset{i}{\rightarrow}$. $\mathcal{R}$ is innermost terminating or innermost normalizing (IN) if every sequence of innermost reduction steps terminates. It is weakly innermost terminating or weakly innermost normalizing (WIN) if for every term $s$ there exists a terminating sequence of innermost reduction steps starting with $s$. By $\infty(s)$ we denote the property that there exists an infinite $(\mathcal{R}$-) derivation starting with $s$. Accordingly, $\neg \infty(s)$ means that every derivation starting with $s$ is finite. By $\infty_{i}(s)$ we denote the property that there exists an infinite innermost derivation starting with $s$. And accordingly, $\neg \infty_{i}(s)$ means that every innermost derivation starting with $s$ is finite.

A partial ordering $>$ on a set $D$ is a transitive and irreflexive binary relation on $D$. A partial ordering $>$ on $\mathcal{T}(\mathcal{F}, \mathcal{V})$ is said to be monotonic (w.r.t. the term structure) if it possesses the replacement property, i.e.

$$
s>t \quad \Longrightarrow \quad C[s]>C[t]
$$

for all $s, t, C[]$. It is stable (w.r.t. substitutions) if

$$
s>t \Longrightarrow \sigma s>\sigma t
$$

for all $s, t, \sigma$. A term ordering on $\mathcal{T}(\mathcal{F}, \mathcal{V})$ is a monotonic and stable partial ordering on $\mathcal{T}(\mathcal{F}, \mathcal{V})$. A reduction ordering is a well-founded term ordering.

A TRS is confluent (CONF) if ${ }^{*} \leftarrow \circ \rightarrow^{*} \subseteq \rightarrow^{*} \circ{ }^{*} \leftarrow$ and locally confluent if $\leftarrow \circ \rightarrow \subseteq \rightarrow^{*} \circ{ }^{*} \leftarrow .^{9}$ A confluent and terminating TRS is said to be convergent or complete $(C O M P)$. If $l_{1} \rightarrow r_{1}, l_{2} \rightarrow r_{2}$ are two rules ${ }^{10}$ of $\mathcal{R}$ and $p$ some non-variable position of $l_{2}$ such that $l_{1}$ and $l_{2} / p$ are unifiable with most general unifier $\sigma$ then $\left(\sigma\left(l_{2}\left[p \leftarrow r_{1}\right]\right), \sigma\left(r_{2}\right)\right)$ is said to be a critical pair $(C P)$ of $\mathcal{R}$ (obtained by overlapping

\footnotetext{
${ }^{8}$ This is a slight abuse of the usual notion of a redex which also comprises the information which rule is applicable. For orthogonal TRSs the corresponding applicable rule is uniquely determined but not in general.

${ }^{9}$ Here, 'o' denotes relation composition.

${ }^{10}$ W.l.o.g. we assume that they do not have common variables.
} 
$l_{1} \rightarrow r_{1}$ with $l_{2} \rightarrow r_{2}$ at position $p$ ). It is well-known that for terminating TRSs local confluence is equivalent to joinability of all critical pairs $(J C P)$. A TRS $\mathcal{R}$ is said to be non-overlapping (NO) if there is no critical pair between rules of $\mathcal{R}$. It is left-linear $(L L)$ if every variable occurs at most once in every left-hand side of an $\mathcal{R}$-rule. $\mathcal{R}$ is orthogonal $(O R T H)^{11}$ if it is left-linear and non-overlapping. It is non-erasing (NE) if $V(r)=V(l)$ for every rule $l \rightarrow r \in \mathcal{R}$. If every critical pair of a TRS $\mathcal{R}$ is obtained by an overlay, i.e. by overlapping left-hand sides of rules at top positions, then $\mathcal{R}$ is said to be an overlay system (OS).

For the sake of readability let us summarize the abbreviating notions defined above which shall be freely used in the sequel. ${ }^{12}$

\section{Abbreviations:}

$\begin{array}{ll}S N & =\text { strongly normalizing (terminating) } \\ W N & =\text { weakly normalizing (weakly terminating) } \\ I N & =\text { innermost normalizing (innermost terminating) } \\ W I N & =\text { weakly innermost normalizing (weakly innermost terminating) } \\ N O & =\text { non-overlapping } \\ L L & =\text { left-linear } \\ O R T H & =\text { orthogonal (non-overlapping and left-linear) } \\ N E & =\text { non-erasing } \\ C P & =\text { critical pair(s) } \\ J C P & =\text { joinability of (all) critical pairs } \\ C O N F & =\text { confluence } \\ C O M P & =\text { completeness (convergence) } \\ O S & =\text { overlay system } \\ \infty(s) & =\text { there exists an infinite derivation starting with s } \\ \infty_{i}(s) & =\text { there exists an infinite innermost derivation starting with s } \\ \neg \infty(s) & =\text { there exists no infinite derivation starting with s } \\ \neg \infty,(s) & =\text { there exists no infinite innermost derivation starting with s }\end{array}$

For properties $P$ and $Q$ of TRSs we write $P+Q$ for denoting the conjunction of $P$ and $Q$. By $P(\mathcal{R})$ we mean that the TRS $\mathcal{R}$ has property $P$. Moreover we also ambiguously use the notation $P(t)$ for terms $t$ provided there is a (sensible) local interpretation for $P(t)$. For instance, $C O N F(t)$ is to denote the property that whenever we have $t \rightarrow^{*} v$ and $t \rightarrow^{*} w$ then there exists a term $s$ with $v \rightarrow^{*} s$ and $w \rightarrow^{*} s$.

\footnotetext{
${ }^{11}$ In the literature this orthogonality property is sometimes called 'regularity'.

${ }^{12}$ These abbreviations are mainly borrowed from [Klo87], [Klo92].

${ }^{13}$ In the sequel we shall prefer 'terminating' instead of 'normalizing' in verbal phrases since it seems to be the more usual notion in literature.
} 


\section{Restricted Termination and Confluence Proper- ties of Term Rewriting Systems}

In the following we shall study under which conditions various restricted kinds of termination imply (uniform) termination (and possibly also confluence under some additional assumptions). Firstly we consider the no-overlap case, i.e. where no critical pairs exist. Then we generalize the analysis by admitting restricted kinds of overlaps. Moreover, where possible, we give examples showing that no precondition of results obtained can be dropped.

It is well-known that under the assumption of termination the confluence property of (finite) TRSs can be easily tested by simply investigating joinability of all critical pairs (cf. [KB70], [Hue80]). If termination is not guaranteed it is in general much more difficult to establish confluence. The problem is that for non-terminating systems joinability of critical pairs is only equivalent to local confluence but not equivalent to confluence any more. There are some sufficient criteria for confluence which do not depend on the termination property but instead on rather strong conditions concerning the syntactical form of the rules and on how joinability of critical pairs has to be possible (see e.g. [Hue80], [Klo92]). Due to the strong preconditions the practical applicability of these criteria is rather limited.

Perhaps the most important and fundamental result concerning confluence of (possibly) non-terminating TRSs is the following: Any orthogonal, i.e. left-linear and nonoverlapping TRS, is confluent (cf. e.g. [Ros73]). This fundamental property is crucial for instance within the field of designing and implementing equational programming languages (cf. e.g. [O'D77], [O'D 85]) and has initiated a couple of investigations about the class of orthogonal TRSs.

In particular, for orthogonal TRSs one also knows some sufficient criteria for termination which are formulated in terms of restricted termination properties (cf. e.g. [O'D77], [Klo92]). But as soon as the orthogonality requirement is weakened, either by allowing critical overlaps or by admitting non-left-linear rules, the main results (at least concerning confluence) do not hold any more, in particular the so-called 'parallel moves lemma' (cf. [Hue80], [Ros73]) which is the technical key lemma for inferring confluence for orthogonal TRSs.

Our overall goal in the following will be to establish (uniform) termination (and confluence) by looking for sufficient conditions involving restricted termination (and confluence) properties.

We shall investigate in the following the termination (and confluence) properties of TRSs which are still non-overlapping but possibly non-left-linear. Later on we shall even relax the no-overlap requirement.

Let us start with the most important known results about confluence and termination properties of orthogonal TRSs. 
Theorem 3.1 (cf. e.g. [Ros73], [O'D77], [Klo92]) Let $\mathcal{R}$ be a TRS with ORTH(R).

Then we have:

(1) $\operatorname{CONF}(\mathcal{R})$.

(2a) $\forall t:[W I N(t) \Longrightarrow I N(t)]$.

(2b) $W I N(\mathcal{R}) \Longrightarrow I N(\mathcal{R})$.

(3a) $\forall t:[I N(t) \Longrightarrow S N(t)]$.

(3b) $I N(\mathcal{R}) \Longrightarrow S N(\mathcal{R})$.

(4) There is no innermost reduction step $s \rightarrow t$ in $\mathcal{R}$ with $\infty(s), \neg \infty(t)$.

(5a) $N E(\mathcal{R}) \Longrightarrow[\forall t:[W N(t) \Longrightarrow S N(t)]]$.

(5b) $N E(\mathcal{R}) \Longrightarrow[W N(\mathcal{R}) \Longrightarrow S N(\mathcal{R})]$.

The following example shows that the left-linearity condition in Theorem 3.1(1) cannot be dropped.

Example 3.2 ([Hue80]) Let $\mathcal{R}$ be the TRS given by the rules

$$
f(x, x) \rightarrow a, \quad f(x, g(x)) \rightarrow b, \quad c \rightarrow g(c) .
$$

Clearly, $\mathcal{R}$ is non-overlapping but neither left-linear nor confluent. We have e.g. the derivations

$$
f(c, c) \rightarrow a \quad \text { and } \quad f(c, c) \rightarrow f(c, g(c)) \rightarrow b .
$$

Here $a$ and $b$ are in normal from. Note that $\mathcal{R}$ is obviously non-terminating and moreover neither weakly innermost terminating nor weakly terminating.

Weak termination is not crucial for the existence of such counterexamples as shown by the following

Example 3.3 (Sivakumar ' 86 ) $^{14}$ Let $\mathcal{R}$ be the TRS given by the rules

$$
f(x, x) \rightarrow g(x), \quad f(x, g(x)) \rightarrow b, \quad h(c, y) \rightarrow f(h(y, c), h(y, y)) .
$$

Clearly, $\mathcal{R}$ is non-overlapping but not confluent. We have e.g. the derivations

$$
h(c, c) \rightarrow f(h(c, c), h(c, c)) \rightarrow f(h(c, c), f(h(c, c), h(c, c))) \rightarrow f(h(c, c), g(h(c, c))) \rightarrow b
$$

and

$$
h(c, c) \rightarrow f(h(c, c), h(c, c)) \rightarrow g(h(c, c)) \rightarrow^{+} g(b) .
$$

Here $b$ and $g(b)$ are in normal from. Note that $\mathcal{R}$ is obviously non-terminating and even not weakly innermost terminating (consider e.g. the term $h(c, c)$ ) but weakly terminating.

\footnotetext{
${ }^{14}$ As mentioned in [DJ90] this example is due to Sivakumar.
} 
In the following we shall consider non-orthogonal TRSs which are still non-overlapping but not necessarily left-linear. Let us start with an easy result about innermost reductions in such systems.

Lemma 3.4 Let $\mathcal{R}$ be a TRS with $N O(\mathcal{R})$. Then we have:

(a) If $s \underset{i}{\longrightarrow}, s \rightarrow u$ then either $t=u$ or there exists a term $v$ with $t \rightarrow v$ and $u \rightarrow v$.

(b) If $s \vec{i}^{m} t, s \vec{i}^{n} u$ then there exists a term $v$ and $m^{\prime} \leq m, n^{\prime} \leq n$ with $t \vec{i}^{n^{\prime}} v$ and $u \rightarrow_{i}^{m^{\prime}} v$.

(c) $\underset{i}{\rightarrow}$ is confluent.

Proof: It suffices to prove (a) since (b) is obtained from (a) by an easy induction, e.g. on $(m, n)$, and (c) is a consequence from (b). Hence let $s \vec{i}_{p} t$ and $s \vec{i}_{q} u$. If the innermost redex positions $p, q$ of $s$ are the same then the applied rule is unique due to $N O(\mathcal{R})$ which implies $t=u$. Otherwise $p$ and $q$ are disjoint and $v$ is uniquely defined by $s \underset{i{ }_{p}}{\rightarrow} \vec{i}_{q} v$ (and $s \vec{i}_{q} u \vec{i}_{p} v$ ).

Our next result shows that for non-overlapping systems the existence of an innermost normal form for some term $t$ implies that any innermost derivation initiated by $t$ is finite.

Lemma 3.5 Let $\mathcal{R}$ be a TRS with $N O(\mathcal{R})$. Then we have:

(a) $\forall t:[W I N(t) \Longrightarrow I N(t)]$.

(b) $W I N(\mathcal{R}) \Longrightarrow I N(\mathcal{R})$.

Proof: It suffices to prove the local version (a) since it implies (b). For a proof by contradiction let $t$ be a term with $W I N(t)$ but not $I N(t)$. Then we know that there exists some innermost derivation

$$
t=t_{0} \underset{i}{\rightarrow} t_{1} \underset{i}{\rightarrow} t_{2} \underset{i}{\rightarrow} \cdots \underset{i}{\rightarrow} t_{n-1} \underset{i}{\rightarrow} t_{n}
$$

with $t_{n}$ irreducible. Obviously we have $\infty_{i}\left(t_{0}\right)$ and $\neg \infty_{i}\left(t_{n}\right)$. Thus there exists some (unique) index $k, 0 \leq k \leq n-1$, with $\infty_{i}\left(t_{k}\right)$ and $\neg \infty_{i}\left(t_{k+1}\right)$. Due to $\infty_{i}\left(t_{k}\right)$ there are terms $t_{k}^{\prime}, t_{k}^{\prime \prime}, \ldots$ such that

$$
t=t_{k} \underset{i}{\rightarrow} t_{k}^{\prime} \underset{i}{\rightarrow} t_{k}^{\prime \prime} \rightarrow \cdots
$$

is an infinite innermost derivation. By applying Lemma 3.4(a) and observing that $t_{k+1} \neq t_{k}^{\prime}$ due to $\infty_{i}\left(t_{k}^{\prime}\right), \neg \infty_{i}\left(t_{k+1}\right)$ we know that there exists a term $t_{k+1}^{\prime}$ with $t_{k}^{\prime} \rightarrow$ $t_{k+1}^{\prime}, t_{k+1} \rightarrow t_{k+1}^{\prime}$ and $\neg \infty_{i}\left(t_{k+1}^{\prime}\right)$. By induction we can conclude that there is an infinite sequence of terms $t_{k+1}^{\prime}, t_{k+1}^{\prime \prime}, \ldots$ such that

$$
t_{k+1} \rightarrow t_{k+1}^{\prime} \rightarrow t_{k+1}^{\prime \prime} \underset{i}{\rightarrow} \cdots
$$


is an infinite innermost derivation. But this is a contradiction to $\neg \infty_{i}\left(t_{k+1}\right)$. Hence we are done.

For the sake of readability we shall use subsequently some more compact notations for special (sequences of) reductions which are introduced now. For parallel (innermost) reduction and normalization (w.r.t. some given TRS $\mathcal{R}$ ) we use the following notations. We write $s \longrightarrow \longrightarrow_{P} t$ if $P$ is a non-empty set of mutually disjoint positions of $s$, and $s \rightarrow^{+} t$ by (parallel) one-step reductions of all the redexes $s / p, p \in P .^{15}$ In particular we write $s \underset{i}{\longrightarrow}{ }_{P} t$ if $s / p$ is an innermost redex of $s$ for all redex positions $p \in P$. We write $s \Downarrow_{P} t$ if $P$ is a non-empty set of mutually disjoint positions of $s$ and $s \rightarrow^{+} t$ by normalizing all the subterms $s / p$ with $p \in P$. In particular we write $s \overleftrightarrow{\longleftrightarrow_{P} t}$ if $t$ is obtained from $s$ by normalizing all the subterms $s / p$ of $s$ with $p \in P$ using only innermost reduction steps. By normalizing a term $t$ we mean reducing it to some normal form. If $W N(t)$ holds then normalization of $t$ is possible but need not yield a unique result. We write $s \longrightarrow \mathbb{\|}, s \stackrel{i}{\longrightarrow} t, s \longrightarrow \Downarrow$ or $s \overleftrightarrow{i} \downarrow t$ if there exists a non-empty set $P$ of mutually disjoint positions of $s$ with $s \longrightarrow\left\|\longrightarrow_{P} t, s \longrightarrow\right\| \longrightarrow_{P} t$, $s \longleftrightarrow_{P} t$ or $s \longleftrightarrow_{P} t$, respectively. Moreover, for the sake of readability we also write $s \longleftrightarrow \bigsqcup_{P}^{<1} t$ if $s=t$ or $s \Downarrow_{P} t$. In the latter case $P$ must clearly be non-empty and $s / p$ must be reducible for some $p \in P$. Analogously, $s \leftrightarrow \longleftrightarrow{ }_{i}^{\leq 1} t$ means $s=t$ or

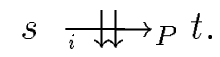

Moreover we shall tacitly make use of the following basic uniqueness properties of parallel reduction and normalization:

$$
N O(\mathcal{R}) \quad \Longrightarrow \quad\left[s\left\|_{P} t_{1} \wedge s\right\|_{P} t_{2} \quad \Longrightarrow \quad t_{1}=t_{2}\right]
$$

and

$$
s \Downarrow_{P} t_{1} \wedge s \Downarrow_{P} t_{2} \wedge \forall p \in P: \operatorname{COMP}(s / p) \Longrightarrow t_{1}=t_{2} .
$$

In a non-overlapping and innermost terminating TRS $\mathcal{R}$ every reduction step can be expressed in terms of (parallel) innermost reduction steps. In slightly generalized form this yields the following.

Lemma 3.6 Let $R$ be a TRS with $N O(\mathcal{R})$ and $s, t$ be terms with $s \rightarrow t$ and $I N(s)$. Then there exist terms $s^{\prime}, t^{\prime}$ with

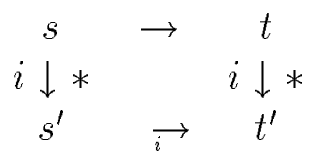

More precisely, we have $s \longrightarrow \downarrow \downarrow \leq 1 \quad s^{\prime} \longrightarrow t^{\prime}$ and $s \rightarrow t \longrightarrow \downarrow \downarrow \leq 1 \quad t^{\prime}$, i.e. $s^{\prime}$ and $t^{\prime}$ are obtained from $s$ and $t$, respectively, by zero or one step(s) of parallel innermost normalization.

\footnotetext{
${ }^{15}$ Note that - for proof-technical reasons which will become clearer later on - we do not require that $s / p$ is a redex for all $p \in P$ but only that at least one subterm $s / p$ of $s$ with $p \in P$ is a redex. This is reflected by the requirement $s \rightarrow^{+} t$.
} 
Proof: Let $\mathcal{R}, s, t$ be given as above with $s \rightarrow_{p, l \rightarrow r} t$. If this is an innermost reduction step we are done. Hence, let us assume that the reduction step $s \rightarrow_{p, l \rightarrow r} t$ is non-innermost. Then we simply perform an innermost reduction of those subterms of $s / p$ to normal form ${ }^{16}$ which correspond to variable positions in the left-hand side of rule $l \rightarrow r$. This may be done by means of parallel reduction and such that subterms corresponding to the same variable in $l$ are innermost reduced to identical normal forms. Let us denote the resulting term by $s^{\prime}$. Then the rule $l \rightarrow r$ is applicable to $s^{\prime}$, too, as an innermost step yielding, let's say, $t^{\prime}$. Clearly, $t^{\prime}$ can also be obtained by innermost reduction steps from $t$, namely by innermost normalizing those subterms in $t / p$ which correspond to variable positions in $r$ in the same way as in $s$.

As an easy consequence of Lemma 3.4 and Lemma 3.6 we obtain the next result.

Lemma 3.7 For any TRS $R$ we have: $N O(\mathcal{R}) \wedge I N(\mathcal{R}) \Longrightarrow \operatorname{CONF}(\mathcal{R})$.

Proof: From Lemma 3.4(c) we know that $\rightarrow$ is confluent. Applying Lemma 3.6 we get $\rightarrow \subseteq \overleftrightarrow{i}^{*}$. From $\vec{i} \subseteq \rightarrow$ it finally follows that $\rightarrow$ is confluent, too.

If we omit the condition $I N(\mathcal{R})$ in the above lemma then $\mathcal{R}$ can be non-confluent (see e.g. Example 3.2 above). Next we shall show that any non-overlapping and innermost terminating TRS is terminating. The following two technical lemmas are useful for giving a shorter proof of this result.

Lemma 3.8 Let $\mathcal{R}$ be given with $N O(\mathcal{R})$ and let $s \rightarrow_{p, l \rightarrow r} t$ be a non-innermost reduction step. Then there exist a set $P$ of mutually distinct positions of s strictly below $p$ and terms $s^{\prime}$, $t^{\prime}$ with $s \underset{i}{\longrightarrow} s_{P} s^{\prime} \rightarrow_{p, l \rightarrow r} t^{\prime}$ and $t \underset{i}{\longrightarrow} \leq^{\prime} t^{\prime}$.

Proof: Let $\mathcal{R}$ be a TRS with $N O(\mathcal{R})$ and $s \rightarrow_{p, l \rightarrow r} t$ be a non-innermost reduction step in $\mathcal{R}$. Obviously we have $s / p=\sigma(l)$ for some substitution $\sigma$. Define $Q_{l}:=\{u \in$ $O(l) \mid l / u \in \mathcal{V}\}$ and $Q_{r}:=\{u \in O(r) \mid r / u \in \mathcal{V}\}$. Since $s \rightarrow_{p, l \rightarrow r} t$ is non-innermost at least one proper subterm of $s / p$ is reducible. From $N O(\mathcal{R})$ we know that all innermost redexes of $s$ strictly below $p$ are below positions $p q$ with $q \in Q_{l}$. This means that we can define $s^{\prime}$ by $s \stackrel{i}{\longrightarrow} P s^{\prime}$ where $P$ is the set of positions of all innermost redexes of $s$ strictly below $p$ and $s^{\prime} / p=\sigma^{\prime}(l)$ for some substitution $\sigma^{\prime}$. Moreover, if $t^{\prime}$ is defined by $s^{\prime} \rightarrow_{p, l \rightarrow r} t^{\prime}$ then we get $s \stackrel{\longrightarrow}{\rightarrow} P s^{\prime} \rightarrow_{p, l \rightarrow r} t^{\prime}$ and $s \rightarrow_{p, l \rightarrow r} t \stackrel{i}{\rightarrow} \frac{<1}{P^{\prime}} t^{\prime}$, where $P^{\prime}$ is the set of positions of all innermost redexes of $t$ strictly below $p$, as desired.

Lemma 3.9 Let $\mathcal{R}$ be given with $N O(\mathcal{R})$ and let $s, t, t^{\prime}$ be terms and $P \subseteq O(s)$ a set of (parallel) positions of $s$ with $s \rightarrow_{p} t, s \underset{i}{\longrightarrow} P s^{\prime}$ such that for at least one $p^{\prime} \in P$ we have $p<p^{\prime}$ and $s / p^{\prime}$ is reducible. Then there exists a term $t^{\prime}$ with $s^{\prime} \rightarrow^{+} t^{\prime}$ and $t \underset{i}{\longrightarrow} \leq 1 t^{\prime}$.

\footnotetext{
${ }^{16}$ Note that the assumption $I N(s)$ of the lemma is crucial for ensuring the existence of an innermost normal form.
} 
Proof: Let $\mathcal{R}$ be given with $N O(\mathcal{R})$ and let $s, t, t^{\prime}$ be terms with $s \rightarrow_{p, l \rightarrow r} t, s \prod_{i} \rightarrow_{P} s^{\prime}$ such that $p<p^{\prime}$ and $s / p^{\prime}$ is reducible for at least one $p^{\prime} \in P$. Hence we know that $s \rightarrow{ }_{p} t$ is not an innermost step. W.l.o.g. we may further assume that $p=\lambda$ (and hence $p<p^{\prime}$ for all $p^{\prime} \in P$ ). From $s \rightarrow_{p} t$ we deduce that $s / p=\sigma(l)$ for some substitution $\sigma$. Now we would like to apply the same rule $l \rightarrow r$ to $s^{\prime} / p$. Remember that $s^{\prime}$ is obtained from $s$ by parallel innermost reduction at the redex positions from $P$. Due to $N O(\mathcal{R})$ all these redex positions are below variable positions of $l$. Thus the only potential reason for non-applicability of $l \rightarrow r$ to $s^{\prime}$ is that $s^{\prime}$ is no longer an instance of $l$ due to the fact that $l \rightarrow r$ might be non-left-linear. But this problem is easily solved by an additional parallel reduction of $s$ at all innermost redex positions (strictly below $p=\lambda$ ) which were not contained in $P$. Let us denote this set of all innermost redex positions of $s$ not contained in $P$ by $Q$. Then we can (uniquely) define terms $s^{\prime \prime}$ and $t^{\prime}$ by $s \underset{i}{\longrightarrow} s_{P} s^{\prime} \underset{i}{\longrightarrow} \stackrel{\leq 1}{Q} s^{\prime \prime} \rightarrow_{p, l \rightarrow r} t^{\prime}$ such that $s \rightarrow_{p, l \rightarrow r} t \underset{i}{\longrightarrow} \leq 1 t^{\prime}$ as desired.

Now we are prepared to prove the first main result.

Theorem 3.10 For any TRS $R$ we have:

(a) $N O(\mathcal{R}) \wedge I N(\mathcal{R}) \Longrightarrow S N(\mathcal{R})$

(b) $N O(\mathcal{R}) \Longrightarrow[\forall t: I N(t) \Longrightarrow S N(t)]$.

Proof: Although we shall prove a more general result later on (cf. Theorem 3.20) we will give a proof here since it is simpler than the one for the more general case.

It suffices to prove (b) which implies (a). For a proof of (b) by contradiction let $\mathcal{R}$ be a TRS with $N O(\mathcal{R})$ and let $t$ be a term with $I N(t)$ but not $S N(t)$. Hence there exists an infinite derivation initiated by $t$. Due to $I N(t)$ every such counterexample contains at least one reduction step which is non-innermost. We consider now a counterexample

$$
D: t=t_{0} \rightarrow t_{1} \rightarrow t_{2} \rightarrow \cdots
$$

which is minimal in the sense that reduction steps are performed at deepest possible positions. More precisely, this means that $D$ has the form

$$
D: t=t_{0} \underset{i}{\rightarrow} t_{1} \underset{i}{\rightarrow} \cdots \underset{i}{\rightarrow} t_{n} \rightarrow_{p} t_{n+1} \rightarrow \cdots
$$

such that $t_{n} \rightarrow_{p} t_{n+1}$ is the first non-innermost step in $D$ and such that

(*) whenever $t_{n} \rightarrow_{q} t_{n}^{\prime}$ for some term $t_{n}^{\prime}$ with $q>p$ then $\neg \infty\left(t_{n}^{\prime}\right) .{ }^{17}$

By applying Lemma 3.8 and using $(*)$ we know that there exist terms $s_{n}, s_{n+1}$ with

$$
t_{n} \stackrel{\mathrm{i}}{\longrightarrow} s_{n} \rightarrow s_{n+1} \quad \text { and } \quad t_{n+1} \stackrel{\longrightarrow}{\rightarrow} \leq^{\leq 1} s_{n+1}
$$

\footnotetext{
${ }^{17}$ Note that in the proof this 'minimality' property is only needed for the first non-innermost step in $D$. Later on, in the proof of the more general Theorem 3.20, we shall make use of the 'full minimality' property.
} 
and $\neg \infty\left(s_{n}\right), \neg \infty\left(s_{n+1}\right)$. Moreover, $\infty\left(t_{n+1}\right)$ implies $t_{n+1} \neq s_{n+1}$, hence $t_{n+1} \nrightarrow s_{n+1}$. In order to obtain a contradiction to $\neg \infty\left(s_{n}\right)$ it suffices to prove that $s_{n} \rightarrow s_{n+1}$ can be extended to an infinite reduction sequence. For that purpose it is sufficient to show that whenever we have $t_{m} \underset{i}{\longrightarrow} s_{m}$ with $\neg \infty\left(s_{m}\right)$ then there exists an index $m^{\prime}>m$ and a term $s_{m^{\prime}}$ with $t_{m^{\prime}} \stackrel{i}{\longrightarrow} s_{m^{\prime}}, s_{m} \rightarrow^{+} s_{m^{\prime}}$ (and $\neg \infty\left(s_{m^{\prime}}\right)$ ). Hence, let $t_{m} \quad \|{ }_{Q} s_{m}$ with $\neg \infty\left(s_{m}\right)$ and $t_{m} \rightarrow{ }_{p} t_{m+1}$. Moreover assume w.l.o.g. that all positions from $Q$ are innermost redex positions of $t_{m}$. We distinguish three cases:

(a) $\forall q \in Q: p \mid q$ : Let $s_{m+1}$ be defined by $s_{m} \rightarrow_{p} s_{m+1}$. Then we get $t_{m} \quad \longrightarrow \longrightarrow Q$ $s_{m} \rightarrow_{p} s_{m+1}$ and $t_{m} \rightarrow t_{m+1} \stackrel{i}{\longrightarrow} s_{Q} s_{m+1}$ with $\neg \infty\left(s_{m+1}\right)$. Hence we can choose $m^{\prime}=m+1$.

(b) $\exists q \in Q: q>p:$ In this case Lemma 3.9 yields the existence of a term $s_{m+1}$ with $s_{m} \rightarrow^{+} s_{m+1}, \neg \infty\left(s_{m+1}\right)$ and $t_{m+1} \quad \| \leq \leq 1 s_{m+1}$. Due to $\infty\left(t_{m+1}\right), \neg \infty\left(s_{m+1}\right)$ we have $t_{m+1} \neq s_{m+1}$, hence $t_{m+1} \stackrel{i}{\longrightarrow} s_{m+1}$. Hence we can choose $m^{\prime}=m+1$.

(c) $\exists q \in Q p \geq q$ : Then we have $p=q$ because $q$ is an innermost redex position of $t_{m}$. From $N O(\mathcal{R})$ we know that reducing $t_{m}$ at position $p=q$ yields a unique result. Thus, choosing $m^{\prime}=m+1$ and $s_{m+1}=s_{m}$ we get $t_{m} \stackrel{i}{\rightarrow} Q s_{m} \rightarrow^{*} s_{m+1}$, $t_{m} \rightarrow{ }_{p} t_{m+1} \underset{i}{ } \longrightarrow \bar{Q}^{\prime} s_{m+1}$ with $Q^{\prime}=Q \backslash\{q\}$. Using $\infty\left(t_{m+1}\right), \neg \infty\left(s_{m+1}\right)$ we can conclude $|Q| \geq 2$ and $t_{m+1} \quad \mathbb{i} \longrightarrow Q^{\prime} s_{m+1}$. Obviously, the reduction $s_{m} \rightarrow^{*} s_{m+1}$ is not a proper one (since we have $s_{m+1}=s_{m}$ ) but we know that after at most $|Q|-1$ steps (in $D$ ) we must be back in case (a) or (b) in which a proper reduction of $s_{m}$ is enabled as desired. ${ }^{18}$

By induction we can finally conclude now that there exists an infinite derivation initiated by $s_{n}$. But this is a contradiction to $\neg \infty\left(s_{n}\right)$. Hence we are done.

As an easy consequence of this result we obtain the following. ${ }^{19}$

Corollary 3.11 Let $\mathcal{R}$ be a TRS. Then the following holds:

$$
N O(\mathcal{R}) \wedge I N(\mathcal{R}) \Longrightarrow \operatorname{COMP}(\mathcal{R})
$$

The next result says that innermost reduction steps in non-overlapping TRSs cannot be critical in the sense that they may destroy the possibility of infinite derivations.

Lemma 3.12 Let $\mathcal{R}$ be a TRS with $N O(\mathcal{R})$. Then there is no innermost reduction step $s \underset{i}{\rightarrow}$ in $\mathcal{R}$ with $\infty(s)$ but $\neg \infty(t)$.

Proof: For a proof by contradiction assume $s \rightarrow t$ with $\infty(s)$ but $\neg \infty(t)$, hence $S N(t)$. Together with $s \rightarrow t$ this implies $W I N(s)$. Using Lemma 3.5 we get $I N(s)$ which by Theorem 3.10 yields $S N(s)$. But this is a contradiction to $\infty(s)$.

\footnotetext{
${ }^{18}$ Formally this last conclusion is proved by an easy induction (on $|Q|$ ).

${ }^{19}$ Note that in Lemma 3.7 we have independently shown the partial result $N O(\mathcal{R}) \wedge I N(\mathcal{R}) \Longrightarrow$ $C O N F(\mathcal{R})$ without making use of $S N(R)$.
} 
Obviously, Lemmas $3.7^{20}, 3.5,3.12$ and Theorem 3.10 express generalizations of Theorem 3.1 (1)-(4). Indeed, it is also possible to prove the following generalization of Theorem 3.1(5).

Theorem 3.13 For any $T R S \mathcal{R}$ the following holds:
(a) $N O(\mathcal{R}) \wedge N E(\mathcal{R}) \wedge W N(\mathcal{R}) \Longrightarrow S N(\mathcal{R})$.
(b) $N O(\mathcal{R}) \wedge N E(\mathcal{R}) \Longrightarrow[\forall t: W N(t) \Longrightarrow S N(t)]$

Proof: It suffices to prove the stronger (b) from which (a) follows easily. For a proof of (b) by contradiction let us assume that $\mathcal{R}$ is a TRS with $N O(\mathcal{R}) \wedge N E(\mathcal{R})$. Moreover, let $t_{0}$ be a term with $W N\left(t_{0}\right)$ but not $S N\left(t_{0}\right)$. Hence there exists a normalizing derivation initiated by $t_{0}$, e.g. a derivation of the form

$$
D: t_{0} \rightarrow t_{1} \rightarrow t_{2} \rightarrow \cdots \rightarrow t_{n-1} \rightarrow t_{n}, n>0
$$

with $t_{n}$ irreducible, hence $\infty\left(t_{0}\right)$ and $\neg \infty\left(t_{n}\right)$. This implies that there is some (unique) index $k, 0 \leq k<n$, with $t_{k} \rightarrow_{p, l \rightarrow r} t_{k+1}, \infty\left(t_{k}\right)$ and $\neg \infty\left(t_{k+1}\right)$. Let us denote the set of variable occurrences of the left- and right-hand side of the applied rule $l \rightarrow r$ by $Q_{l}:=\{q \in O(l) \mid l / q \in \mathcal{V}\}$ and $Q_{r}:=\{q \in O(r) \mid r / q \in \mathcal{V}\}$, respectively. By Lemma 3.12 we know that $t / p$ must be a non-innermost redex of $t$. Since $\mathcal{R}$ is non-overlapping $t_{k} / p q$ must be reducible for at least one $q \in Q_{l}$. From $N E(\mathcal{R})$ we know moreover that $\left\{t_{k} / p q \mid q \in Q_{l}\right\}=\left\{t_{k+1} / p q \mid q \in Q_{r}\right\}$. Furthermore $\neg \infty\left(t_{k+1}\right)$ implies $S N\left(t_{k+1} / p q\right)$ for all $q \in Q_{r}$ which - due to $N O(\mathcal{R})$ - yields $C O M P(t)$ for all $t \in\left\{t_{k} / p q \mid q \in Q_{l}\right\}=$ $\left\{t_{k+1} / p q \mid q \in Q_{r}\right\}$. Hence, for $P:=\left\{p q \mid q \in Q_{l}\right\}, P^{\prime}:=\left\{p q \mid q \in Q_{r}\right\}$ there exist (uniquely defined) terms $s_{k}, s_{k+1}$ with $t_{k} \uplus_{P} s_{k} \rightarrow_{p, l \rightarrow r} s_{k+1}, t_{k} \rightarrow_{p, l \rightarrow r} t_{k+1} \longleftrightarrow_{P^{\prime}}$ $s_{k+1}$ such that $\infty\left(t_{k}\right), \neg \infty\left(t_{k+1}\right), \neg \infty\left(s_{k+1}\right)$. Since parallel normalization of $t_{k}$ at all positions from $P$ can be achieved by using only innermost steps we obtain $t_{k} \vec{i}^{+} s_{k+1}$ with $\infty\left(t_{k}\right)$ and $\neg \infty\left(s_{k+1}\right)$. This implies $W I N\left(t_{k}\right)$ which yields $I N\left(t_{k}\right)$ by Lemma 3.5 and $S N\left(t_{k}\right)$ by Theorem 3.10. But this is a contradiction to $\infty\left(t_{k}\right)$.

Note that the assumption $N E(\mathcal{R})$ above cannot be dropped. To wit consider

Example 3.14 $\mathcal{R}:=\{a \rightarrow f(a), f(x) \rightarrow b\}$ is clearly non-overlapping and weakly terminating but not strongly terminating and also not weakly innermost terminating. For instance, $b$ is a normal form of $f(a)$ but cannot be obtained by innermost reduction.

In view of Lemmas 3.4, 3.5, and Theorem 3.10 one might be tempted to conjecture that instead of requiring $N O(\mathcal{R})$ and $I N(\mathcal{R})$ it should also be sufficient to require $J C P(\mathcal{R})$ and $W I N(\mathcal{R})$ for guaranteeing $S N(\mathcal{R})$ and $C O N F(\mathcal{R})$. But this is not sufficient as witnessed by

Example 3.15 For the TRS $\mathcal{R}$ given by $a \leftarrow b \leftrightarrow c \rightarrow d^{21}$ we have $J C P(\mathcal{R})$ and $W I N(\mathcal{R})$ but neither $C O N F(\mathcal{R})$ nor $S N(\mathcal{R})$.

\footnotetext{
${ }^{20}$ Lemma 3.7 generalizes Theorem 3.1(1) only in the sense that instead of $(N O+L L)(\mathcal{R})$ it also suffices to have $(N O+I N)(\mathcal{R})$ for inferring $C O N F(\mathcal{R})$.

${ }^{21}$ Of course, $b \leftrightarrow c$ is to denote both rules $b \rightarrow c$ and $c \rightarrow b$ (and not the disjunction $b \rightarrow c$ or $c \rightarrow b$ ).
} 
Even the stronger requirement $J C P(\mathcal{R})$ and $I N(\mathcal{R})$ is not sufficient for ensuring $S N(\mathcal{R})$ and $C O N F(\mathcal{R})$ as can be seen from

Example 3.16 Let $\mathcal{R}$ be given by $a \leftarrow f(b) \leftrightarrow f(c) \rightarrow d, b \rightarrow e, c \rightarrow e^{\prime}, f(e) \rightarrow a$ and $f\left(e^{\prime}\right) \rightarrow d$. Here it is easily verified that $J C P(\mathcal{R})$ and $I N(\mathcal{R})$ hold but neither $\operatorname{CONF}(\mathcal{R})$ nor $S N(\mathcal{R})$.

Another possibly tempting conjecture might be to insist on $I N(\mathcal{R})$ and even require $C O N F(\mathcal{R})$ in order to infer $S N(\mathcal{R})$. But this is also not true in general.

Example 3.17 Consider the TRS $\mathcal{R}$ given by $f(a) \rightarrow f(a), a \rightarrow b$ for which we have $\operatorname{CONF}(\mathcal{R})$ and $I N(\mathcal{R})$ but not $S N(\mathcal{R})$.

But a common feature of the latter two counterexamples consists in the fact that for both systems critical pairs were constructed by overlaps strictly below the root. This is crucial as will be shown next.

To this end let us reconsider now Theorem 3.10. Essentially, what we have done there is to drop the precondition $L L(\mathcal{R})$ in Theorem 3.1(3), i.e. to require only $N O+I N$ instead of $N O+L L+I N$ for deriving $S N(+C O N F)$. We shall strengthen this result now by proving that the rather restrictive property $N O$ may be further weakened by restricting possible overlaps and guaranteeing local confluence. To be precise, $N O$ is replaced by $O S+J C P$, i.e. corresponding TRSs have to be overlay systems (i.e. critical pairs are admitted but only on top level) which are locally confluent (i.e. all critical pairs are joinable).

In order to enable a simpler proof of this main result we need the following two auxiliary lemmas.

Lemma 3.18 Let $\mathcal{R}$ be a TRS with $O S(\mathcal{R})$ and $J C P(\mathcal{R})$. Moreover let $s \rightarrow_{p, l \rightarrow r} t$ be a non-innermost reduction step with $S N(\bar{s})$ for all proper subterms $\bar{s}$ of $s / p$. Then there exist terms $s^{\prime}, t^{\prime}$ and sets $P \subseteq O(s), Q \subseteq O(t)$ such that

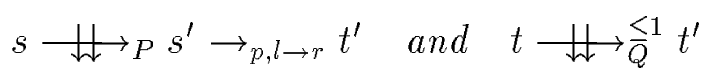

with $p<p^{\prime}$ for all $p^{\prime} \in P, C O M P(s / u)$ for all $u \in P$ and $C O M P(t / v)$ for all $v \in Q$.

Proof: Under the assumptions of the lemma let $Q_{l}$ and $Q_{r}$ be the sets of variable positions of $l$ and $r$, respectively, and define $P:=\left\{p q \mid q \in Q_{l}\right\}, Q:=\left\{p q \mid q \in Q_{r}\right\}$. By assumption we know $S N(\bar{s})$ for all proper subterms $\bar{s}$ of $s / p$. Due to $J C P(\mathcal{R})$ this implies $C O M P(\bar{s})$ for all proper subterms $\bar{s}$ of $s / p$. From $O S(\mathcal{R})$ and the fact that the step $s \rightarrow_{p, l \rightarrow r} t$ is non-innermost we conclude that at least one subterm $s / u$ of $s$, $u \in P$, is reducible. Thus $s^{\prime}$ defined by $s \Downarrow_{P} s^{\prime}$ exists (and is unique). Since $s^{\prime}$ is an instance of $l, t^{\prime}$ is also uniquely defined by $s^{\prime} \rightarrow_{p, l \rightarrow r} t^{\prime}$. Moreover, $t^{\prime}$ can be obtained from $t$ by (uniquely) normalizing all subterms $t / u$ of $t$ with $u \in Q$, e.g. $t \longrightarrow \downarrow \backslash 1$. Finally, $\{t / v \mid v \in Q\} \subseteq\{s / u \mid u \in P\}$ implies $C O M P(t / v)$ for all $v \in Q$ as desired.

The next result is a technical key lemma which will be used below in the proof of Theorem 3.20 for properly extending some given finite derivation to an infinite one. 
Lemma 3.19 Let $\mathcal{R}$ be a TRS with $O S(\mathcal{R})$ and $J C P(\mathcal{R})$. Moreover let $s \rightarrow_{p, l \rightarrow r} t$ and $s \longrightarrow \longleftrightarrow_{U} s^{\prime}$ with $\operatorname{COMP}(s / u)$ for all $u \in U, C O M P(s / v)$ for all $v \in O(s)$ with $v>p, U_{>p}:=\{u \in U \mid u>p\} \neq \emptyset$ and $s / u$ reducible for all $u \in U_{>p}$. Then there exists a term $t^{\prime}$ and $W \subseteq O(t)$ such that $s \Downarrow_{U} s^{\prime} \rightarrow^{+} t^{\prime}$ and $t \longleftrightarrow_{W}^{<1} t^{\prime}$ with $C O M P(t / w)$ for all $w \in W$.

Proof: Let $\mathcal{R}, s, t, s^{\prime}, p, l \rightarrow r$ and $U$ be given as above. W.l.o.g. we may assume $p=\lambda$ which implies $u>\lambda$ for all $u \in U$ (due to the assumption $U_{>p}:=\{u \in U \mid u>$ $p\} \neq \emptyset)$. Define $Q_{l}:=\{q \in O(l) \mid l / q \in \mathcal{V}\} \subseteq O(s), Q_{r}:=\{q \in O(r) \mid r / q \in \mathcal{V}\} \subseteq O(t)$. Due to $O S(\mathcal{R})$ we know that for every redex $s / q$ of $s$ with $q>\lambda$ we have $q \geq q^{\prime}$ for some $q^{\prime} \in Q_{l}$. Hence, every $u \in U$ is below or above some $q \in Q_{l}$. Now we define $s^{\prime \prime}$ by $s \longleftrightarrow Q_{l} s^{\prime \prime}, t^{\prime}$ by $t \longrightarrow{ }_{\bar{Q}_{r}} t^{\prime}$ and show that $s \Downarrow_{U} s^{\prime} \Downarrow_{\bar{Q}_{l}}^{\leq 1} s^{\prime \prime} \rightarrow_{p, l \rightarrow r} t^{\prime}$ holds (by appropiately rearranging certain reduction sequences). To this end we consider all those positions from $U \cup P_{l}$ which are minimal among $U \cup Q_{l}$ w.r.t. $\leq$.

- If $q \in Q_{l}$ is minimal among $U \cup Q_{l}$ such that $u_{1}, \ldots, u_{m}, m \geq 1$, are all positions from $U$ below $q$ then due to $\operatorname{COMP}(s / q)$ (since $q>\lambda$ ) normalization of $s$ at $p$ can be achieved by normalizing $s$ at $u_{1}, \ldots, u_{m}$ followed by normalizing the resulting term at $q$.

- If $u \in U$ is strictly minimal among $U \cup Q_{l}$ such that $q_{1}, \ldots, q_{n}$ are all positions from $Q_{l}$ strictly below $u$ then normalization of $s$ at $u$ can be achieved by first normalizing $s$ at $q_{1}, \ldots, q_{n}$ yielding let's say $\hat{s}$ and then normalizing in $\hat{s}$ the subterm $h a t(s) / u$. But the latter normalization must be empty, i.e. $\hat{s} / u$ must be irreducible. To see this, let us assume that $\hat{s} / u$ is reducible, let's say at position $v$ with rule $l_{1} \rightarrow r_{1}$ and matching substitution $\sigma_{1}$, i.e. $\sigma_{1}\left(l_{1}\right)=\hat{s} / u v$. By the construction of $\hat{s}$ we know that $\hat{s} / q_{i}$ is irreducible for $i=1, \ldots, n$. Hence, $u v>\lambda$ (which follows from $u>\lambda$ ) is a non-variable position of both $\hat{s}$ and $l$. Moreover we know by the construction of $\hat{s}$ that $\hat{s} / u=\sigma^{\prime}(l) / u$ for some substitution $\sigma^{\prime}$ which implies $\sigma^{\prime}(l) / u v=\sigma_{1}\left(l_{1}\right)$. But this means that there exists a critical pair between the rules $l_{1} \rightarrow r_{1}$ and $l \rightarrow r$ which is not a critical overlay due to $u v>\lambda$. Hence we have a contradiction to $O S(\mathcal{R})$.

In summary this means that we have $s \Downarrow_{U} s^{\prime} \rightarrow^{*} s^{\prime \prime} \rightarrow_{p, l \rightarrow r} t^{\prime}$ and $s \rightarrow_{p, l \rightarrow r} t \dashv \downarrow$ $\longrightarrow \bar{Q}_{r}^{\leq 1} t^{\prime}$ with $C O M P(t / w)$ for all $w \in Q_{r}$ due to $\left\{t / w \mid w \in Q_{r}\right\} \subseteq\left\{s / v \mid v \in Q_{l}\right\}$ and $\operatorname{COMP}(s / v)$ for all $v \in O(s)$ with $v>\lambda$. Hence choosing $W:=Q_{r}$ we are done.

Now we are prepared to state and prove the following main result.

Theorem 3.20 For any TRS $\mathcal{R}$ we have:

(a) $O S(\mathcal{R}) \wedge J C P(\mathcal{R}) \wedge I N(\mathcal{R}) \Longrightarrow S N(\mathcal{R}) \wedge C O N F(\mathcal{R})$, and

(b) $O S(\mathcal{R}) \wedge J C P(\mathcal{R}) \Longrightarrow[\forall s:[I N(s) \Longrightarrow S N(s) \wedge C O N F(s)]]$,

i.e. any locally confluent and innermost terminating overlay system is terminating and confluent (part (a)) which also holds in the localized version (b). 
Proof: It suffices to prove (b) since (a) follows from it. For a proof of (b) by contradiction let $\mathcal{R}$ be a TRS with the assumed properties $O S(\mathcal{R})$ and $J C P(\mathcal{R})$ and let $t_{0}$ be a term with $I N\left(t_{0}\right)$ but not $S N\left(t_{0}\right)$. Then we consider a 'minimal' counterexample for $t_{0}$, i.e. an infinite derivation

$$
D: \quad t_{0} \rightarrow t_{1} \rightarrow t_{2} \rightarrow \cdots
$$

satisfying

(1) $\forall j \geq 0: \infty\left(t_{j}\right)$, and

(2) $\forall j \geq 0 \forall s_{j}:\left[t_{j} \rightarrow_{p} t_{j+1} \wedge t_{j} \rightarrow_{q} s_{j}\right.$ with $\left.q>p\right] \Longrightarrow \neg \infty\left(s_{j}\right)$.

The minimality assumption (2) says that reduction steps are performed at deepest possible positions. This means in particular that innermost reduction steps are preferred as long as possible. Due to $I N\left(t_{0}\right)$ there must exist some first non-innermost step in $D$, let's say $t_{n} \rightarrow_{p, l \rightarrow r} t_{n+1}$. The minimality assumption (2) implies $S N(t)$ and hence $C O M P(t)$ (due to $J C P(\mathcal{R})$ ) for every proper subterm $t$ of $t_{n} / p$. By applying Lemma 3.18 and using (2) we know that there exist (uniquely defined) terms $s_{n}, s_{n+1}$ and $P \subseteq O\left(t_{n}\right), p>p^{\prime}$ for all $p^{\prime} \in P \neq \emptyset, Q \subseteq O\left(t_{n+1}\right)$ with $t_{n} \uplus_{P} s_{n} \rightarrow_{p, l \rightarrow r} s_{n+1}$ and $t_{n+1} \longleftrightarrow{ }_{Q}^{\leq 1} s_{n+1}$ such that $\neg \infty\left(s_{n}\right)$ and $\operatorname{COMP}\left(t_{n+1} / q\right)$ for all $q \in Q$. Moreover, $\infty\left(t_{n+1}\right)$ and $\neg \infty\left(s_{n+1}\right)$ imply $t_{n+1} \neq s_{n+1}$, hence $t_{n+1} \longleftrightarrow_{Q} s_{n+1}$.

In order to obtain a contradiction to $\neg \infty\left(s_{n}\right)$, it suffices to prove that $s_{n} \rightarrow s_{n+1}$ can be extended to an infinite reduction sequence. For that purpose it is sufficient to show that

whenever we have $t_{m} \Downarrow_{U} s_{m}$ with $\infty\left(t_{m}\right), \neg \infty\left(s_{m}\right)$ and $\operatorname{COMP}\left(t_{m} / u\right)$ for all $u \in U$

then there exists an index $m^{\prime}>m$, a term $s_{m^{\prime}}$ and a set $U^{\prime} \subseteq O\left(t_{m^{\prime}}\right)$ with $s_{m} \rightarrow^{+} s_{m^{\prime}}, t_{m^{\prime}} \uplus_{U^{\prime}} s_{m^{\prime}}, \neg \infty\left(s_{m^{\prime}}\right)^{22}$ and $\operatorname{COMP}\left(t_{m^{\prime}} / u\right)$ for all $u \in U^{\prime}$.

Hence, let $t_{m} \longrightarrow{ }_{U} s_{m}$ with $\infty\left(t_{m}\right)$ and $t_{m} \rightarrow_{p, l \rightarrow r} t_{m+1}$ (for arbitrary $m>n$ ) and assume w.l.o.g. that $t_{m} / u$ is reducible for all $u \in U$. Then we have to distinguish the following three cases:

(a) $\forall u \in U: u \mid p:$ In this case we can choose $m^{\prime}=m+1$ and $s_{m^{\prime}}=s_{m+1}$ is obtained from $s_{m}$ by applying $l \rightarrow r$ at position $p$, i.e. we get $t_{m} \Downarrow_{U} s_{m} \rightarrow_{p, l \rightarrow r} s_{m+1}$, $t_{m} \rightarrow_{p, l \rightarrow r} t_{m+1} \longleftrightarrow s_{m+1}$ with $\neg \infty\left(s_{m+1}\right)$ and $C O M P\left(t_{m+1} / u\right)$ for all $u \in U$ as desired.

(b) $\exists u \in U: u>p:$ From the minimality assumption (2) we know $\operatorname{COMP}\left(t_{m} / q\right)$ (due to $J C P(\mathcal{R})$ ) for all $q>p, q \in O\left(t_{m}\right)$. Hence, applying Lemma 3.19 we get a term $s_{m+1}$ and a set $W \subseteq O\left(t_{m+1}\right)$ with $s_{m} \rightarrow^{+} s_{m+1}, t_{m+1} \longrightarrow \downarrow \downarrow \leq 1$ $s_{m+1}, \neg \infty\left(s_{m+1}\right)$ and $C O M P\left(t_{m+1} / w\right)$ for all $w \in W$. Moreover, $\neg \infty\left(s_{m+1}\right)$ and $\infty\left(t_{m+1}\right)$ imply $t_{m+1} \neq s_{m+1}$, hence $t_{m+1} \longleftrightarrow{ }_{W} s_{m+1}$ as desired. 
(c) $\exists u \in U: p \geq u$ : In this case $s_{m+1}$ is defined by $t_{m} \rightarrow_{p, l \rightarrow r} t_{m+1} \longrightarrow \stackrel{\leq 1}{\longrightarrow} s_{m+1} \cdot{ }^{23}$ From $t_{m} \longleftrightarrow_{U} s_{m}, C O M P\left(t_{m} / u\right)$ for all $u \in U$ and $p \geq u$ for some $u \in U$ we get $C O M P\left(t_{m+1} / u\right)$ for all $u \in U$, and $s_{m}=s_{m+1}$. Moreover, $\neg \infty\left(s_{m}\right)$ implies $\neg \infty\left(s_{m+1}\right)$ which together with $\infty\left(t_{m+1}\right)$ yields $t_{m+1} \neq s_{m+1}$, hence $t_{m+1} \longleftrightarrow{ }_{U}$ $s_{m+1}$. The only problem now is that the reduction sequence passing by $s_{m}$ is not properly extended due to $s_{m}=s_{m+1}$. But from $C O M P\left(t_{m} / u\right)$ for all $u \in U$ we know that only finitely many subsequent steps in $D$ can take place below positions from $U$. Hence, eventually case (a) or case (b) applies again in which a proper extension of the reduction sequence passing by $s_{m}$ is possible as desired. ${ }^{24}$

By induction we can conclude that there exists an infinite derivation starting from $s_{n}$. But this is a contradiction to $\neg \infty\left(s_{n}\right)$. Hence we are done.

Note that for proving Theorem 3.20 we cannot apply the (simpler) construction used for proving Theorem 3.10 by means of parallel (unique) one-step reduction. The crucial point is that reduction of some term $t$ at some position $p$ need not be unique since critical overlays are allowed. But - as we have shown - it is possible to modify the construction by performing parallel normalization steps instead of parallel reduction steps.

Note moreover that we cannot weaken the precondition of Theorem 3.20 by omitting the requirement $J C P(\mathcal{R})$. To wit, consider

Example 3.21 ${ }^{25}$ Let $\mathcal{R}$ be the TRS consisting of the rules

$$
f(a, b, x) \rightarrow f(x, x, x), \quad G(x, y) \rightarrow x, \quad G(x, y) \rightarrow y
$$

Obviously, $\mathcal{R}$ is an innermost terminating overlay system but it is not strongly normalizing as can be seen from the infinite (cyclic) derivation

$$
\begin{aligned}
f(a, b, G(a, b)) & \rightarrow f(G(a, b), G(a, b), G(a, b)) \\
& \rightarrow f(a, G(a, b), G(a, b)) \\
& \rightarrow f(a, b, G(a, b)) \\
& \rightarrow \cdots .
\end{aligned}
$$

Moreover, omitting the condition $O S(\mathcal{R})$ in Theorem 3.20 is not possible, either. Consider e.g. ${ }^{26}$

\footnotetext{
${ }^{22}$ Note that this is an implicit consequence of $s_{m} \rightarrow^{+} s_{m^{\prime}}$ and $m^{\prime}>m$, hence it could be omitted here.

${ }^{23}$ More precisely, the definition of $s_{m+1}$ depends on whether $t_{m+1} / u$ is reducible for at least one $u \in U$. In this case $s_{m+1}$ is defined by $t_{m+1} \Downarrow_{U} s_{m+1}$. Otherwise, i.e. if $t_{m+1} / u$ is irreducible for all $u \in U$ then we define $s_{m+1}:=t_{m+1}$.

${ }^{24}$ Formally, this can be proved by an additional easy induction.

${ }^{25}$ Note that by partitioning these 3 rules into $\mathcal{R}_{1}:=\{f(a, b, x) \rightarrow f(x, x, x)\}$ and $\mathcal{R}_{2}:=\{G(x, y) \rightarrow$ $x, G(x, y) \rightarrow y\}$ we obtain Toyama's counterexample to modularity of termination (cf. [Toy87a]).

${ }^{26}$ See also Example 3.16 which is already a counterexample to this conjecture.
} 
Example $3.22{ }^{27}$ Let $\mathcal{R}$ be the TRS consisting of the rules

$$
f(a, b, x) \rightarrow f(x, x, x), a \rightarrow c, b \rightarrow c, f(x, y, z) \rightarrow c, G(x, y, y) \rightarrow x, G(y, x, y) \rightarrow x .
$$

Here, $\mathcal{R}$ is innermost terminating and all critical pairs are joinable (the system is even confluent) but there are two critical pairs which are no critical overlays and $\mathcal{R}$ is non-terminating as witnessed e.g. by

$$
\begin{aligned}
f(a, b, G(a, b, b)) & \rightarrow f(G(a, b, b), G(a, b, b), G(a, b, b)) \\
& \rightarrow f(a, G(a, b, b), G(a, b, b)) \\
& \rightarrow f(a, G(c, b, b), G(a, b, b)) \\
& \rightarrow f(a, G(c, b, c), G(a, b, b)) \\
& \rightarrow f(a, b, G(a, b, b)) \\
& \rightarrow \cdots
\end{aligned}
$$

According to Theorem 3.1(3) $(N O+L L+I N)$ implies $S N$, too. Theorem 3.10 says that even $(N O+I N)$ implies $S N$. Moreover, from Theorem 3.20 we know that the weaker property $(O S+J C P+I N)$ implies $S N$, too. In view of these results another interesting conjecture would be the following:

$$
(C O N F+L L+I N) \Longrightarrow S N \text {. }
$$

But again this is not true in general.

Example 3.23 (Example 3.17 continued) Let $\mathcal{R}$ be given by the rules $f(a) \rightarrow f(a)$ and $a \rightarrow b$. Obviously, this system is confluent, left-linear and innermost normalizing but is clearly non-terminating.

By weakening the no-overlap requirement of Lemmas 3.5, 3.12 and Theorem 3.13 in a manner analogous to Theorem 3.20 one might be tempted to state the following conjectures which would be generalizations of Theorem 3.1(2), (4) and (5).

$$
\begin{aligned}
& O S(\mathcal{R}) \wedge J C P(\mathcal{R}) \Longrightarrow \quad[W I N(\mathcal{R}) \Longrightarrow I N(\mathcal{R})] \\
& O S(\mathcal{R}) \wedge J C P(\mathcal{R}) \Longrightarrow \quad \begin{array}{l}
\text { there is no innermost reduction step } s \rightarrow t \\
\text { in } \mathcal{R} \text { with } \infty(s) \text { and } \neg \infty(t), \text { and }
\end{array} \\
& O S(\mathcal{R}) \wedge J C P(\mathcal{R}) \wedge N E(\mathcal{R}) \Longrightarrow[W N(\mathcal{R}) \Longrightarrow S N(\mathcal{R})] .
\end{aligned}
$$

But $(C 1),(C 2)$ and $(C 3)$ are all refuted by the following very simple counterexample.

Example 3.24 Let $\mathcal{R}$ consist of the two rules $a \rightarrow a$ and $a \rightarrow b$. Clearly, $\mathcal{R}$ is a nonerasing overlay system where the only critical pair is a joinable overlay. Moreover, every term has a normal form that can be computed by innermost reduction, but obviously $\mathcal{R}$ is not innermost terminating and hence not strongly terminating, too. Furthermore we have $a \rightarrow b$ with $\infty(a)$ and $\neg \infty(b)$.

\footnotetext{
${ }^{27}$ Note that by partitioning these rules into $\mathcal{R}_{1}:=\{f(a, b, x) \rightarrow f(x, x, x), a \rightarrow c, b \rightarrow c, f(x, y, z) \rightarrow$ $c\}$ and $\mathcal{R}_{2}:=\{G(x, y, y) \rightarrow x, G(y, x, y) \rightarrow x\}$ we obtain a counterexample to modularity of completeness (cf. [Dro89]).
} 


\section{Applications to Disjoint and Non-Disjoint Unions of TRSs}

We shall now consider modular properties of TRSs and apply our previous results in order to derive some interesting new results and provide simplified proofs of known ones. Let us first give a brief (and incomplete) overview of known results in this field. Modular properties of term rewriting systems, i.e. properties which are preserved under disjoint unions, have attracted an increasing attention within the last few years. From a theoretical point of view and also for efficiency reasons it is very useful to know whether a combined TRS has some property whenever this property already holds for the single 'modules'. A simple and natural way of such 'modular' constructions is given by the concept of 'direct sum' ([Toy87b]) or 'disjoint union'. ${ }^{28}$ Two TRSs $\mathcal{R}_{1}$ and $\mathcal{R}_{2}$ over disjoint signatures $\mathcal{F}_{1}$ and $\mathcal{F}_{2}$, respectively, are said to be disjoint if $\mathcal{F}_{1}$ and $\mathcal{F}_{2}$ are disjoint, i.e. $\mathcal{F}_{1} \cap \mathcal{F}_{2}=\emptyset$ (in that case $\mathcal{R}_{1}$ and $\mathcal{R}_{2}$ are necessarily disjoint, too). The (disjoint) union of two disjoint TRSs $\mathcal{R}_{1}, \mathcal{R}_{2}$ is denoted by $\mathcal{R}_{1} \oplus \mathcal{R}_{2}$. We shall also speak of the disjoint union of $\mathcal{R}_{1}$ and $\mathcal{R}_{2}$ using the implicit convention that $\mathcal{R}_{1}$ and $\mathcal{R}_{2}$ are assumed to be disjoint TRSs. A property $\mathrm{P}$ of TRSs is said to be modular if the following holds for all disjoint TRSs $\mathcal{R}_{1}, \mathcal{R}_{2}: \mathcal{R}_{1} \oplus \mathcal{R}_{2}$ has property $P$ iff both $\mathcal{R}_{1}$ and $\mathcal{R}_{2}$ have property $P .{ }^{29}$

Toyama [Toy $87 \mathrm{~b}$ ] has shown that confluence is modular. The termination property, however, is in general not modular as witnessed by the following counterexample of [Toy87b] (cf. Example 3.21) :

Example 4.1 Consider the TRSs given by

$\mathcal{R}_{1}: \quad f(a, b, x) \rightarrow f(x, x, x) \quad$ and $\quad \mathcal{R}_{2}: \quad G(x, y) \rightarrow x$

Clearly, both $R_{1}$ and $R_{2}$ are terminating, but $\mathcal{R}_{1} \oplus \mathcal{R}_{2}$ admits e.g. the following infinite derivation:

$$
\begin{array}{rll}
f(a, b, G(a, b)) & \rightarrow \mathcal{R}_{1} & f(G(a, b), G(a, b), G(a, b)) \\
& \rightarrow \mathcal{R}_{2} & f(a, G(a, b), G(a, b)) \\
& \rightarrow \mathcal{R}_{2} & f(a, b, G(a, b)) \\
& \rightarrow \mathcal{R}_{1} & \cdots
\end{array}
$$

Note, that in this example $\mathcal{R}_{2}$ is not confluent. Other, more complicated examples by Klop \& Barendregt as well as by Toyama gathered in [Toy $87 \mathrm{a}$ ] show that $\mathcal{R}_{1} \oplus \mathcal{R}_{2}$ may be non-terminating even if $R_{1}$ and $R_{2}$ are both terminating, confluent and interreduced.

All these counterexamples have some common feature. Namely, one of the systems contains a duplicating rule, ${ }^{30}$ i.e. a rule $l \rightarrow r$ where some variable occurs strictly more

\footnotetext{
${ }^{28}$ Roughly spoken, the concept of 'direct sum' as defined in [Toy $\left.87 \mathrm{~b}\right]$ is slightly more general than that of 'disjoint union' because it allows for renaming function symbols in order to obtain disjointness.

${ }^{29}$ Later on we shall also consider invariance properties of combination mechanisms which are more general than 'direct sum modularity'.

${ }^{30}$ Such a TRS is said to be duplicating.
} 
often in $\mathrm{r}$ than in 1 , and the other system contains a collapsing rule $l^{\prime} \rightarrow r^{\prime}$, i.e. $r^{\prime}$ is a variable ${ }^{31}$. As proved in [Rus87] termination is modular for the class of collapse-free TRSs as well as for the class of non-duplicating systems. In [Mid89] it is shown that the disjoint union $\mathcal{R}_{1} \oplus \mathcal{R}_{2}$ of two terminating TRSs $\mathcal{R}_{1}, \mathcal{R}_{2}$ is again terminating whenever one of the systems is non-duplicating and collapse-free. Moreover, as shown in [KO90a], simple termination is a modular property of (finite) TRSs, i.e. whenever two (finite) TRSs $\mathcal{R}_{1}, \mathcal{R}_{2}$ can be shown to be terminating by means of simplification orderings then this holds for their disjoint union, too. A unified approach to modular termination of rewriting is provided in [Gra91], [Gra92a], [Gra93] where still more general sufficient conditions for modularity of termination are presented.

Some extensions and generalizations of these known results on modular termination to the case of conditional TRSs as well as to some restricted classes of non-disjoint unions of TRSs can be found in [Mid90], [KO90b], [Mid93], [Gra92c], [Gra93] and [Oh193].

An interesting result not subsumed by other ones is due to [TKB89] where it is shown that completeness is modular for left-linear TRSs.

The main link between our abstract results proved in the previous section and the problem of modular termination of rewriting is provided by the following easy but fundamental result.

Lemma 4.2 Innermost termination (IN) is a modular property of TRSs.

Proof: Let $\mathcal{R}_{1}^{\mathcal{F}_{1}}, \mathcal{R}_{2}^{\mathcal{F}_{2}}$ be disjoint innermost terminating TRSs. Then we show by structural induction that $I N(t)$ holds for all $t \in \mathcal{T}\left(\mathcal{F}_{1} \uplus \mathcal{F}_{2}, \mathcal{V}\right)$. If $t$ is a variable then $I N(t)$ is trivially satisfied. If $t$ is a constant of $\mathcal{F}_{1} \uplus \mathcal{F}_{2}$ then $I N(t)$ is satisfied by assumption. If $t=f\left(t_{1}, \ldots, t_{n}\right)$ then we have by induction hypothesis $I N\left(t_{1}\right) \wedge$ $\ldots \wedge I N\left(t_{n}\right)$. W.l.o.g. we may further assume $f \in \mathcal{F}_{1}$. Now, if $t$ is irreducible (w.r.t. $\mathcal{R}_{1} \uplus \mathcal{R}_{2}$ ) we are done. Otherwise, we know by induction hypothesis that for every $i \in\{1, \ldots, n\}$ every innermost derivation of $t_{i}$ is eventually terminating. This means that every innermost derivation starting with $t$ is either terminating or has the form $t=f\left(t_{1}, \ldots, t_{n}\right) \rightarrow_{i}^{*} f\left(t_{1}^{\prime}, \ldots, t_{n}^{\prime}\right) \rightarrow_{\lambda} \ldots$ where $t_{1}^{\prime}, \ldots, t_{n}^{\prime}$ are all irreducible (w.r.t. $\left.\mathcal{R}_{1} \uplus \mathcal{R}_{2}\right)$. If in the latter case $t^{\prime}:=f\left(t_{1}^{\prime}, \ldots, t_{n}^{\prime}\right)$ were not innermost normalizing, i.e. $\infty_{i}\left(t^{\prime}\right)$, we could conclude that $\mathcal{R}_{1}$ is not innermost normalizing (this is easily seen by replacing every maximal subterm $s_{1}, \ldots, s_{m}$ of $t$ with $\operatorname{root}\left(s_{i}\right) \in \mathcal{F}_{2}$ by a fresh variable $x_{i}$ such that $x_{j}=x_{k}$ whenever $s_{j}=s_{k}$ ).

Note that weak normalization and weak innermost normalization are modular properties of TRSs, too, as shown in [BKM89], [Dro89] and [KK90].

Combining Lemma 4.2 with Theorem 3.10 we obtain

Theorem 4.3 Termination (and hence completeness) is modular for the class of nonoverlapping TRSs.

\footnotetext{
${ }^{31} \mathrm{~A}$ system without collapsing rules is said to be collapse-free.
} 
Proof: Let $\mathcal{R}_{1}, \mathcal{R}_{2}$ be two non-overlapping and terminating TRSs. Hence we have $(N O+S N)\left(\mathcal{R}_{1}\right)$ and $(N O+S N)\left(\mathcal{R}_{2}\right)$ implying $(N O+I N)\left(\mathcal{R}_{1}\right)$ and $(N O+I N)\left(\mathcal{R}_{2}\right)$. Since $N O$ is obviously modular and $I N$, too, due to Lemma 4.2 we conclude $(N O+$ $I N)\left(\mathcal{R}_{1} \oplus \mathcal{R}_{2}\right)$. By applying Theorem 3.10 we finally obtain $(N O+S N)\left(\mathcal{R}_{1} \oplus \mathcal{R}_{2}\right)$ and hence $(S N+C O N F)\left(\mathcal{R}_{1} \oplus \mathcal{R}_{2}\right)$ as desired.

Similarly, Theorem 3.20(a) yields the following generalized modularity result.

Theorem 4.4 Termination (and hence completeness) is modular for the class of locally confluent overlay systems.

Proof: Analagous to the proof of Theorem 4.3 using Lemma 4.2 and Theorem 3.20(a).

Note that this result is in a sense a variation of the main result of [TKB89] which says that $(L L+C O N F+S N)$ is modular. Theorem 4.4 states that the left-linearity restriction can be dropped if a stronger kind of confluence property is satisfied, namely, every critical pair must be a joinable overlay. In other words, $(O S+J C P+S N)$ is modular.

Moreover, Example 4.1 shows that dropping $J C P$ is not possible because in general $(O S+S N)$ is not modular.

Furthermore let us show now that Theorem 3.20(a) can be used to give a substantially simplified proof of the main result from [MT91]. For a precise formulation we need some terminology from [MT91].

Definition 4.5 (cf. [MT91], [KO90b]) Let $\mathcal{R}^{\mathcal{F}}$ be a TRS with $\mathcal{F}=\mathcal{C} \uplus \mathcal{D}$ such that $\mathcal{D}=\left\{\operatorname{root}(l) \mid l \rightarrow r \in \mathcal{R}^{\mathcal{F}}\right\}$ and $\mathcal{C}=\mathcal{F} \backslash \mathcal{D}$. The symbols in $\mathcal{D}$ are said to be defined symbols and those in $\mathcal{C}$ constructors. To emphasize the partitioning of $\mathcal{F}$ into $\mathcal{F}=\mathcal{C} \uplus \mathcal{D}$ we also write $\left(\mathcal{R}^{\mathcal{F}}, \mathcal{C}, \mathcal{D}\right)$ or $(\mathcal{R}, \mathcal{C}, \mathcal{D})$ instead of $\mathcal{R}^{\mathcal{F}}$ and say that $\mathcal{R}^{\mathcal{F}}=(\mathcal{R}, \mathcal{C}, \mathcal{D})$ is a TRS with constructors. Then $\mathcal{R}^{\mathcal{F}}$ is said to be a constructor system (CS) if $\mathcal{C} \supseteq \bigcup_{f\left(t_{1}, \ldots, t_{n_{i}}\right) \rightarrow r_{i} \in \mathcal{R}^{\mathcal{F}}} \bigcup_{j=1}^{n_{i}} F\left(t_{j}\right),{ }^{32}$ i.e. if no left-hand side argument contains a defined symbol. ${ }^{33}$ If $\left(\mathcal{R}_{1}^{\mathcal{F}_{1}}, \mathcal{C}_{1}, \mathcal{D}_{1}\right),\left(\mathcal{R}_{2}^{\mathcal{F}_{2}}, \mathcal{C}_{2}, \mathcal{D}_{2}\right)$ are constructor systems with $\mathcal{D}_{1} \cap \mathcal{F}_{2}=\mathcal{D}_{1} \cap$ $\left(\mathcal{C}_{2} \uplus \mathcal{D}_{2}\right)=\emptyset=\mathcal{D}_{2} \cap\left(\mathcal{C}_{1} \uplus \mathcal{D}_{1}\right)=\mathcal{D}_{2} \cap \mathcal{F}_{1}$ then $\left(\mathcal{R}_{1} \cup \mathcal{R}_{2}\right)^{\mathcal{F}_{1} \cup \mathcal{F}_{2}}=\left(\mathcal{R}_{1} \cup \mathcal{R}_{2}, \mathcal{C}_{1} \cup \mathcal{C}_{2}, \mathcal{D}_{1} \uplus \mathcal{D}_{2}\right)$ is said to be a combination of constructor systems (with disjoint sets of defined symbols and common constructors).

Middeldorp and Toyama have shown in [MT91] that completeness is preserved for combinations of constructor systems. In fact, a slightly more general result is proved in [MT91].

\footnotetext{
${ }^{32}$ Note that function symbols from $\mathcal{F}$ which do not occur in rules of $\mathcal{R}^{\mathcal{F}}$ are (by definition) considered to be constructors.

${ }^{33}$ This definition of constructor system corresponds to what is usually meant when one speaks of a constructor discipline (for specifying functions).
} 
Since any constructor system is by definition a special overlay system we easily obtain from Theorem 3.20 the following

Corollary 4.6 For any $T R S \mathcal{R}$ we have: $C S(\mathcal{R}) \wedge I N(\mathcal{R}) \wedge J C P(\mathcal{R}) \Longrightarrow$ $S N(\mathcal{R}) \wedge C O N F(\mathcal{R})$, i.e. any innermost terminating and locally confluent constructor system is terminating and confluent, hence complete.

Moreover we need the following result.

Lemma 4.7 The combination of innermost terminating constructor systems (with deisjoint sets of defined symbols) is again an innermost terminating constructor system.

Proof: The proof is very similar to that of Lemma 4.2 and uses similar arguments as given in [MT91] in the proof of the fact that weak normalization is preserved under the union of constructor systems with disjoint sets of defined symbols.

Let $(\mathcal{R}, \mathcal{C}, \mathcal{D})=\left(\left(\mathcal{R}_{1} \cup \mathcal{R}_{2}\right)^{\mathcal{F}_{1} \cup \mathcal{F}_{2}}, \mathcal{C}_{1} \cup \mathcal{C}_{2}, \mathcal{D}_{1} \uplus \mathcal{D}_{2}\right)$ be the combination of two innermost normalizing $\operatorname{CSs}\left(\mathcal{R}_{1}^{\mathcal{F}_{1}}, \mathcal{C}_{1}, \mathcal{D}_{1}\right)$ and $\left(\mathcal{R}_{2}^{\mathcal{F}_{2}}, \mathcal{C}_{2}, \mathcal{D}_{2}\right)$. We will show by structural induction that for every term $t \in \mathcal{T}(\mathcal{C} \uplus \mathcal{D}, \mathcal{V})$ we have $I N(t)$. The case $t \in \mathcal{C} \uplus \mathcal{D} \uplus \mathcal{V}$, i.e. if $t$ is a constant or a variable, is easy. Now suppose $t=f\left(t_{1}, \ldots, t_{n}\right)$ and assume by induction hypothesis $I N\left(t_{1}\right) \wedge \ldots \wedge I N\left(t_{n}\right)$. If $f$ is a constructor, i.e. $f \in \mathcal{C}$ then $I N(t)$ follows from $I N\left(t_{1}\right) \wedge \ldots \wedge I N\left(t_{n}\right)$. Else we may assume w.l.o.g. $f \in \mathcal{D}_{1}$. Clearly, every innermost derivation issued by $t$ is terminating or has the form

$$
t=f\left(t_{1}, \ldots, t_{n}\right) \rightarrow_{i}^{*} f\left(t_{1}^{\prime}, \ldots, t_{n}^{\prime}\right) \underset{i}{\rightarrow_{\lambda}} \cdots
$$

with $t_{j}^{\prime}$ irreducible (w.r.t. $\left.\mathcal{R}_{1} \cup \mathcal{R}_{2}\right), 1 \leq j \leq n$. If in the latter case $t^{\prime}:=f\left(t_{1}^{\prime}, \ldots, t_{n}^{\prime}\right)$ were not innermost normalizing, we could conclude that $\mathcal{R}_{1}$ is not innermost normalizing, i.e. $\infty_{i}\left(t^{\prime}\right)$ (w.r.t. $\mathcal{R}_{1}$ ). This is easily seen by replacing every maximal subterm $s_{1}, \ldots, s_{m}$ of $t$ with $\operatorname{root}\left(s_{i}\right) \in \mathcal{D}_{2}$ by a fresh variable $x_{i}$ such that $x_{j}=x_{k}$ whenever $s_{j}=s_{k}$.

Now it is easy to give a substantially simplified and 'modular' proof of

Theorem 4.8 ([MT91]) The combination of complete constructor systems (with disjoint sets of defined function symbols) is again complete. ${ }^{34}$

Proof: Straightforward by combining corollary 4.6 and Lemma 4.7.

Theorem 3.20 provides us with a rather general approach for ensuring the invariance of completeness under certain combinations of TRSs. What is necessary to apply this

\footnotetext{
${ }^{34}$ One may allow here common defined function symbols under the additional assumption that for such symbols the corresponding definition rules have to coincide in both systems. See [MT91] for more details and a precise definition of 'composable' constructor systems and 'decomposable' properties of constructor systems.
} 
result is to define appropriate classes of (not necessarily disjoint) TRSs and/or combination mechanisms which ensure that the properties $O S, J C P$ and $I N$ are preserved. Above we have seen that this is easily possible for combinations of constructor systems (with disjoint sets of defined symbols). Moreover it is straightforward to cover the more general case of combined systems with shared constructors.

Definition 4.9 (cf. [KO90b]) Let $\mathcal{R}_{1}^{\mathcal{F}_{1}}=\left(\mathcal{R}_{1}, \mathcal{C}_{1}, \mathcal{D}_{1}\right), \mathcal{R}_{2}^{\mathcal{F}_{2}}=\left(\mathcal{R}_{2}, \mathcal{C}_{2}, \mathcal{D}_{2}\right)$ be TRSs with constructors such that $\mathcal{D}_{1} \cap \mathcal{D}_{2}=\mathcal{D}_{1} \cap \mathcal{C}_{2}=\mathcal{D}_{2} \cap \mathcal{C}_{1}=\emptyset$. Then the TRS $\mathcal{R}^{\mathcal{F}}=(\mathcal{R}, \mathcal{C}, \mathcal{D})=\left(\mathcal{R}_{1} \cup \mathcal{R}_{2}, \mathcal{C}_{1} \cup \mathcal{C}_{2}, \mathcal{D}_{1} \uplus \mathcal{D}_{2}\right)$ is said to be a combined system with shared constructors ( $\mathcal{C}$ and disjoint sets $\mathcal{D}_{1}, \mathcal{D}_{2}$ of defined symbols).

Clearly, every combination of constructor systems (with disjoint sets of defined symbols) is a combined system with shared constructors but not vice-versa in general, since for the latter defined symbols may occur in left-hand side arguments which is not allowed for constructor systems.

It is easy to see that if $\mathcal{R}^{\mathcal{F}}=\left(\mathcal{R}_{1} \cup \mathcal{R}_{2}\right)^{\mathcal{F}_{1} \cup \mathcal{F}_{2}}=\left(\mathcal{R}_{1} \cup \mathcal{R}_{2}, \mathcal{C}_{1} \cup \mathcal{C}_{2}, \mathcal{D}_{1} \uplus \mathcal{D}_{2}\right)$ is a combined system with shared constructors such that $\mathcal{R}_{1}, \mathcal{R}_{2}$ are overlay systems then the combined system $\mathcal{R}^{\mathcal{F}}$ is an overlay system, too. Moreover we also have $C P(\mathcal{R})=$ $C P\left(\mathcal{R}_{1}\right) \cup C P\left(\mathcal{R}_{2}\right)$, hence local confluence of $\mathcal{R}$ is inherited from local confluence of $\mathcal{R}_{1}, \mathcal{R}_{2}$. Furthermore innermost termination of $\mathcal{R}$ is also inherited from innermost termination of $\mathcal{R}_{1}, \mathcal{R}_{2}$ as shown next.

Lemma 4.10 Let $\mathcal{R}^{\mathcal{F}}=\left(\mathcal{R}_{1} \cup \mathcal{R}_{2}\right)^{\mathcal{F}_{1} \cup \mathcal{F}_{2}}$ be a combined system with shared constructors. Then we have:

$$
I N\left(\mathcal{R}_{1}^{\mathcal{F}_{1}}\right) \wedge I N\left(\mathcal{R}_{2}^{\mathcal{F}_{2}}\right) \quad \Longleftrightarrow \quad I N\left(\mathcal{R}^{\mathcal{F}}\right)
$$

Proof: The 'if'-direction is trivial and the 'only-if'-direction is completely analogous to the proof of Lemma 4.7 .

With the above observation, the preceding lemma and Theorem 3.20 we obtain the following result as corollary.

Theorem 4.11 Let $\mathcal{R}^{\mathcal{F}}=\left(\mathcal{R}_{1} \cup \mathcal{R}_{2}\right)^{\mathcal{F}_{1} \cup \mathcal{F}_{2}}$ be a combined system with shared constructors. Then $\mathcal{R}^{\mathcal{F}}$ is a complete overlay system if and only if both $\mathcal{R}_{1}^{\mathcal{F}_{1}}$ and $\mathcal{R}_{2}^{\mathcal{F}_{2}}$ are complete overlay systems.

Even more generally we shall consider now certain kinds of hierarchical combinations of TRSs and show that our main Theorem 3.20 is applicable here, too. Hierarchical combinations are particularly interesting from a practical point of view, for instance in many rewriting based function definition formalisms. For illustration let us consider a simple example.

Example 4.12 Consider the TRSs (CSs) given by

$$
\begin{aligned}
\mathcal{R}_{1}: & 0+y & \rightarrow y & \mathcal{F}_{1}=\mathcal{D}_{1} \uplus \mathcal{C}_{1}, \\
& s(x)+y & \rightarrow s(x+y) & \mathcal{C}_{1}=\{0, s\}, \mathcal{D}_{1}=\{+\}
\end{aligned}
$$




$$
\begin{array}{rlll}
\mathcal{R}_{2}: & & & \mathcal{F}_{2}=\mathcal{D}_{2} \uplus \mathcal{C}_{2}, \\
s(x) * y & \rightarrow x * y+y & & \mathcal{C}_{2}=\{0, s,+\}, \mathcal{D}_{2}=\{*\}
\end{array}
$$

where 0 and $s$ (uccessor) are common constructors. Here the combined system $\mathcal{R}^{\mathcal{F}}=$ $\left(\mathcal{R}_{1} \cup \mathcal{R}_{2}\right)^{\mathcal{F}_{1} \cup \mathcal{F}_{2}}$ neither is a combination of constructor systems (with disjoint sets of defined symbols) nor a combined system with shared constructors. But $\mathcal{R}^{\mathcal{F}}$ is a hierarchical combination of constructor systems in the sense that defined function symbols of the base system $\mathcal{R}_{1}$ may occur in right-hand sides of $\mathcal{R}_{2}$ but do not occur in left-hand sides of $\mathcal{R}_{2}$. In other words, the function '*' is defined recursively by $\mathcal{R}_{2}$ in terms of the predefined function '+' of the base system $\mathcal{R}_{1}$.

Formally we get the following

Definition 4.13 Let $\mathcal{R}_{1}^{\mathcal{F}_{1}}=\left(\mathcal{R}_{1}^{\mathcal{F}_{1}}, \mathcal{C}_{1}, \mathcal{D}_{1}\right), \mathcal{R}_{2}^{\mathcal{F}_{2}}=\left(\mathcal{R}_{2}^{\mathcal{F}_{2}}, \mathcal{C}_{2}, \mathcal{D}_{2}\right)$ be TRSs with constructors such that $\mathcal{D}_{2} \cap\left(\mathcal{C}_{1} \uplus \mathcal{D}_{1}\right)=\emptyset$. Moreover, defining $\mathcal{P}_{2}:=\mathcal{C}_{2} \cap \mathcal{D}_{1}$ to be the set of predefined symbols (of $\mathcal{R}_{2}^{\mathcal{F}_{2}}$ ) we require that no predefined symbol from $\mathcal{P}_{2}$ occurs in a left-hand side of $\mathcal{R}_{2}$. In other words, defined symbols of $\mathcal{R}_{1}^{\mathcal{F}_{1}}$ may be used as predefined (constructor) symbols in $\mathcal{R}_{2}^{\mathcal{F}_{2}}$ but only on right-hand sides. Then the TRS $\left(\mathcal{R}_{1} \cup \mathcal{R}_{2}\right)^{\mathcal{F}_{1} \cup \mathcal{F}_{2}}$ is said to be the hierarchical combination of (the base system) $\mathcal{R}_{1}$ with the (non-base system) $\mathcal{R}_{2} \cdot{ }^{35}$

For such cases we would still like to be able to infer termination (and confluence) of the hierarchical combination $\mathcal{R}_{1} \cup \mathcal{R}_{2}$ from possibly restricted termination (and confluence) properties of $\mathcal{R}_{1}, \mathcal{R}_{2}$. The inheritance of local confluence is easily obtained for the case that the combination does not give rise to new critical pairs. Formally we get the following.

Definition 4.14 (cf. [Mid90]) Two TRSs $\mathcal{R}_{1}, \mathcal{R}_{2}$ are said to be non-interfering if $C P\left(\mathcal{R}_{1} \cup \mathcal{R}_{2}\right)=C P\left(\mathcal{R}_{1}\right) \cup C P\left(\mathcal{R}_{2}\right)$.

Lemma 4.15 (cf. [Mid90]) For non-interfering TRSs $\mathcal{R}_{1}, \mathcal{R}_{2}$ we have:

$$
J C P\left(\mathcal{R}_{1}\right) \wedge J C P\left(\mathcal{R}_{2}\right) \quad \Longrightarrow \quad J C P\left(\mathcal{R}_{1} \cup \mathcal{R}_{2}\right)
$$

i.e., $\mathcal{R}_{1} \cup \mathcal{R}_{2}$ is locally confluent if both $\mathcal{R}_{1}$ and $\mathcal{R}_{2}$ are locally confluent.

One easily verifiable criterion for non-interference of TRSs is the following.

Lemma 4.16 Let $\mathcal{R}_{1}^{\mathcal{F}_{1}}, \mathcal{R}_{2}^{\mathcal{F}_{2}}$ be TRSs with $\operatorname{root}\left(\right.$ lhs $\left.\left(\mathcal{R}_{1}\right)\right) \cap F\left(\right.$ lhs $\left.\left(\mathcal{R}_{2}\right)\right)=\operatorname{root}\left(\operatorname{lhs}\left(\mathcal{R}_{2}\right)\right) \cap$ $F\left(\right.$ lhs $\left.\left(\mathcal{R}_{1}\right)\right)=\emptyset$ where lhs $(\mathcal{R})$ denotes the set of left-hand sides of a TRS $\mathcal{R}, F(t)$ $(F(T))$ the set of function symbols occurring in a term $t$ (or a set $T$ of terms) and $\operatorname{root}(t)(\operatorname{root}(T))$ the set of root symbols of a term $t$ (or a set $T$ of terms). Then $\mathcal{R}_{1}$, $\mathcal{R}_{2}$ are non-interfering, hence: $J C P\left(\mathcal{R}_{1}\right) \wedge J C P\left(\mathcal{R}_{2}\right) \Longrightarrow J C P\left(\mathcal{R}_{1} \cup \mathcal{R}_{2}\right)$.

\footnotetext{
${ }^{35}$ Note that both $\mathcal{R}_{1}^{\mathcal{F}_{1}}$ and $\mathcal{R}_{2}^{\mathcal{F}_{2}}$ are TRSs with constructors, but not necessarily constructor systems $(\mathrm{CSs})$.
} 
The following examples demonstrate some subtleties of hierarchical combinations of TRSs.

Example 4.17 (Example 3.2 continued) Consider the TRSs given by

$$
\mathcal{R}_{1}: f(x, x) \rightarrow a, f(x, g(x)) \rightarrow b \quad \text { and } \quad \mathcal{R}_{2}: c \rightarrow g(c)
$$

Here, both systems are confluent but their hierarchical combination ${ }^{36}$ is only locally confluent and not confluent any more.

Example 4.18 ([Gra91]) Consider the TRSs given by

$$
\mathcal{R}_{1}: a \rightarrow b \quad \text { and } \quad \mathcal{R}_{2}: h(x, x) \rightarrow h(a, b)
$$

Here, both systems are confluent and terminating but their hierarchical combination is only confluent but not terminating any more (consider e.g. the infinite cyclic derivation $h(b, b) \rightarrow h(a, b) \rightarrow h(b, b) \cdots)$. Note that $a \in \mathcal{D}_{1}=\mathcal{P}_{2}$ occurs on the right-hand side of the $\mathcal{R}_{1}$-rule $h(x, x) \rightarrow h(a, b)$ below $h \in \mathcal{D}_{2}$.

By modifying Example 4.17 above we can show that both termination and confluence may be lost under hierarchical combinations.

Example 4.19 Consider the TRSs given by

$$
\mathcal{R}_{1}: f(x, x) \rightarrow a, f(x, g(x)) \rightarrow b, d \rightarrow c \quad \text { and } \quad \mathcal{R}_{2}: e(c) \rightarrow g(e(d))
$$

with $\mathcal{C}=\{a, b, c, g\}, \mathcal{D}_{1}=\{f, d\}, \mathcal{D}_{2}=\{e\}$ and $\mathcal{P}_{2}=\{d\}$. Here, both systems are confluent and terminating but their hierarchical combination $\mathcal{R}=\mathcal{R}_{1} \cup \mathcal{R}_{2}$ is neither confluent nor terminating. We have e.g. $f(e(c), e(c)) \rightarrow a$ and $f(e(c), e(c)) \rightarrow^{+} b$ in $\mathcal{R}$, where both $a$ and $b$ are irreducible. Moreover, $e(c) \rightarrow g(e(d)) \rightarrow g(e(c)) \rightarrow \ldots$ is an infinite (looping) derivation in $\mathcal{R}$. Note again that in $\mathcal{R}_{2}$ the predefined symbol $d \in \mathcal{D}_{1}$ occurs below $e \in \mathcal{D}_{2}$ in the right-hand side of $e(c) \rightarrow g(e(d))$.

The following examples show that for a hierarchical combination of $\mathcal{R}_{1}$ with $\mathcal{R}_{2}$ termination can get lost if defined symbols are allowed to be nested on left or right-hand sides of $\mathcal{R}_{1}$ or $\mathcal{R}_{2}$.

Example 4.20 ${ }^{37}$ Consider the TRSs given by

$$
\begin{array}{rlrl}
\mathcal{R}_{1}: f(a, b, x) & \rightarrow f(x, x, x) & \mathcal{R}_{2}: g(x, y, y) & \rightarrow x \\
f(x, y, z) & \rightarrow c & g(y, y, x) & \rightarrow x \\
a & \rightarrow c & \\
b & \rightarrow c &
\end{array}
$$

\footnotetext{
${ }^{36}$ Note that the combination is not properly hierarchical since no defined symbol from $\mathcal{R}_{1}$ is used in right-hand sides of $\mathcal{R}_{2}$. We have here $\mathcal{D}_{1}=\{f\}, \mathcal{D}_{2}=\{c\}$ and $\mathcal{P}_{2}=\emptyset$. In particular, $g$ is a common constructor.

37This example which shows that completeness is not a modular property of TRSs is due to [Dro89].
} 
where $\mathcal{D}_{1}=\{a, b, f\}, \mathcal{D}_{2}=\{g\}, \mathcal{C}=\{c\}$ and $\mathcal{P}_{2}=\emptyset .{ }^{38}$ Both $\mathcal{R}_{1}$ and $\mathcal{R}_{2}$ are easily seen to be terminating (and confluent) but $\mathcal{R}=\mathcal{R}_{1} \cup \mathcal{R}_{2}$ is non-terminating. We have e.g. an infinite (cyclic) derivation of the form $f(a, b, g(a, b, b)) \rightarrow^{+} f(a, b, g(a, b, b)) \rightarrow \ldots$ The system $\mathcal{R}$ even is a disjoint combination of TRSs. Obviously, the first $\mathcal{R}_{1}$-rule contains nested $\mathcal{D}_{1}$-symbols on its left-hand side.

Example 4.21 (cf. [Kri92]) Consider the hierarchical combination of TRSs given by

$$
\mathcal{R}_{1}: f(x) \rightarrow x \quad \text { and } \quad \mathcal{R}_{2}: g(x) \rightarrow f(x), h(a) \rightarrow h(g(a))
$$

with $\mathcal{D}_{1}=\{f\}, \mathcal{D}_{2}=\{g, h\}, \mathcal{P}_{2}=\{f\}$ and $\mathcal{C}=\{a\}$. Again both systems are clearly terminating but their hierarchical combination $\mathcal{R}=\mathcal{R}_{1} \cup \mathcal{R}_{2}$ is non-terminating. We have for instance the following infinite (cyclic) derivaion in $\mathcal{R}: h(a) \rightarrow_{\mathcal{R}_{2}} h(g(a)) \rightarrow \mathcal{R}_{2}$ $h(f(a)) \rightarrow \mathcal{R}_{1} h(a) \rightarrow \cdots$. Here, the right-hand side of the second rule of $\mathcal{R}_{2}$ contains nested $\mathcal{D}_{2}$-symbols, namely $h$ and $g$.

In order to identify (more) principal problems concerning the invariance of termination (and confluence) under hierarchical combinations we consider now a (simplified) schematic version of Example 4.12 .

Example 4.22 Assume that $\mathcal{R}_{1}^{\mathcal{F}_{1}}$ is some complete $C S$ with a set $\mathcal{C}=\{0, s\}$ of constructors where some (unary) functions $g$ and $h$ are (pre)defined, i.e. $\mathcal{D}_{1}=\{g, h\}$ such that $\mathcal{R}_{1}$ is a complete constructor system. Then we consider the hierarchical combination $\mathcal{R}^{\mathcal{F}}=\left(\mathcal{R}_{1} \cup \mathcal{R}_{2}\right)^{\mathcal{F}_{1} \cup \mathcal{F}_{2}}$ where $\mathcal{R}_{2}^{\mathcal{F}_{2}}$ is given by $\begin{aligned} f(0) & =t_{0} \\ f(s(x)) & =g(f(h(x)))\end{aligned}$ with $t_{0}$ some fixed term from $\mathcal{T}(\mathcal{C})$ and $\mathcal{D}_{2}=\{f\}$. It is obvious that $\mathcal{R}_{2}$ is also complete, but the interesting question is whether the combined system $\mathcal{R}^{\mathcal{F}}$ is terminating (and confluent). Intuitively it is clear that the definition of $g$ provided in $\mathcal{R}_{1}$ is irrelevant for the termination behaviour of $f$ which only depends on the definition of $h$. For instance, if for $h$ interpreted over the natural numbers we have ' $h(x) \leq x$ ' for all $x$ then the definition of $f$, i.e. the combined system, is terminating (and confluent, hence complete). If however ' $h(x)>x$ ' for all $x$ then the definition of $f$ is non-terminating in the combined system.

Hence, in order to obtain a sufficient criterion for the invariance of termination under such hierarchical combinations we try to solve the above problem by eliminating the possibility that predefined function symbols may occur on right-hand sides of the nonbase system below function symbols from $\mathcal{D}_{2}$. The above considerations motivate the following definitions (cf. Dershowitz [Der92]).

Definition 4.23 Let $\mathcal{R}^{\mathcal{F}}=\left(\mathcal{R}_{1} \cup \mathcal{R}_{2}\right)^{\mathcal{F}_{1} \cup \mathcal{F}_{2}}$ with $\mathcal{F}_{1}=\mathcal{C} \uplus \mathcal{D}_{1}, \mathcal{F}_{2}=\left(\mathcal{C} \uplus \mathcal{P}_{2}\right) \uplus \mathcal{D}_{2}{ }^{39}$ be the hierarchical combination of $\mathcal{R}_{1}^{\mathcal{F}_{1}}$ with $\mathcal{R}_{2}^{\mathcal{F}_{2}}$. Then we say that $\mathcal{R}_{2}$ is flat if symbols

\footnotetext{
${ }^{38}$ Hence, the combination is not only hierarchical but even modular.

${ }^{39}$ Note that we assume here w.l.o.g. that the set of constructor symbols of $\mathcal{R}_{2}^{\mathcal{F}_{2}}$ consists of the constructor symbols of $\mathcal{R}_{1}^{\mathcal{F}_{1}}$ plus the predefined symbols from $\mathcal{D}_{1}$ (for a given hierarchical combination of $\mathcal{R}_{1}^{\mathcal{F}_{1}}=\left(\mathcal{R}_{1}, \mathcal{C}_{1}, \mathcal{D}_{1}\right)$ with $\mathcal{R}_{2}^{\mathcal{F}_{2}}=\left(\mathcal{R}_{2}, \mathcal{C}_{2}, \mathcal{D}_{2}\right)$ this can always be achieved by taking $\mathcal{C}$ to be $\mathcal{C}:=$ $\mathcal{C}_{1} \cup\left(\mathcal{C}_{2} \backslash \mathcal{D}_{1}\right)$ and considering $\left(\mathcal{R}_{1}, \mathcal{C}, \mathcal{D}_{1}\right)$ and $\left(\mathcal{R}_{2}, \mathcal{C}, \mathcal{D}_{2}\right)$ instead of $\mathcal{R}_{1}^{\mathcal{F}_{1}}$ and $\mathcal{R}_{2}^{\mathcal{F}_{2}}$, respectively.
} 
from $\mathcal{D}_{2}$ are not nested, on both sides of the rules of $\mathcal{R}_{2}$ (that is, no path from the root symbol to a constant or variable has more than one $\mathcal{D}_{2}$ symbol along it). ${ }^{40}$ In this case the combined system $\mathcal{R}^{\mathcal{F}}$ is also said to be flat. Moreover the hierarchical combination $\mathcal{R}^{\mathcal{F}}$ is said to be separated if no predefined function symbol from $\mathcal{D}_{1}$ occurs below a defined one of $\mathcal{D}_{2}$ on a right-hand side of $\mathcal{R}_{2}$.

Now we are prepared to state the following interesting result.

Theorem 4.24 A flat and separated hierarchical combination $\mathcal{R}^{\mathcal{F}}=\left(\mathcal{R}_{1} \cup \mathcal{R}_{2}\right)^{\mathcal{F}_{1} \cup \mathcal{F}_{2}}$ is innermost normalizing if and only if both $\mathcal{R}_{1}^{\mathcal{F}_{1}}$ and $\mathcal{R}_{2}^{\mathcal{F}_{2}}$ are innermost normalizing.

Proof: The 'only-if' direction of the theorem is trivial. Hence let us assume that $\mathcal{R}^{\mathcal{F}}=\left(\mathcal{R}_{1} \cup \mathcal{R}_{2}\right)^{\mathcal{F}_{1} \cup \mathcal{F}_{2}}$ is a flat and separated hierarchical combination such that both $\mathcal{R}_{1}$ and $\mathcal{R}_{2}$ are innermost normalizing. Let $\mathcal{D}_{1}$ and $\mathcal{D}_{2}$ be the set of defined symbols of $\mathcal{R}_{1}$ and $\mathcal{R}_{2}$, respectively, $\mathcal{C}$ be the set of common constructors and $\mathcal{P}_{2} \subseteq \mathcal{D}_{1}$ be the set of predefined function symbols of $\mathcal{R}_{2}$. Assume now for a proof by contradiction that $\mathcal{R}$ is not innermost normalizing. Then there exists a counterexample, i.e. an infinite innermost $\mathcal{R}$-derivation

$$
D: t_{0} \underset{i}{\rightarrow} t_{1} \underset{i}{\rightarrow} t_{2} \underset{i}{\longrightarrow} t_{3} \underset{i}{\longrightarrow} \cdots
$$

W.l.o.g. we may further assume that all proper subterms of $t_{0}$ are irreducible (if there exists an infinite innermost $\mathcal{R}$-derivation then this derivation either contains a root reduction step or else, using the pigeonhole principle, one can extract from it another infinite $\mathcal{R}$-derivation starting with a root reduction step). We distinguish the following three cases according to where the root symbol of $t_{0}$ does stem from and show that in each case we obtain a contradiction.

(a) If $\operatorname{root}\left(t_{0}\right) \in \mathcal{C}$ then $t_{0}$ is obviously $\mathcal{R}$-irreducible contradicting the assumption $\infty_{i}\left(t_{0}\right)$.

(b) If $\operatorname{root}\left(t_{0}\right) \in \mathcal{D}_{1}$ we know that $t_{0}$ has the form $t_{0}=C\left[s_{1}, \ldots, s_{n}\right]$ where $C[, \ldots$, is a context containing only symbols from $\mathcal{C} \cup \mathcal{D}_{1} \cup \mathcal{V}$ and $s_{1}, \ldots, s_{n}$ are all the maximal subterms of $t_{0}$ with root symbol in $\mathcal{D}_{2}$. The first step of $D$ above then has the form

$$
t_{0}=C\left[s_{1}, \ldots, s_{n}\right] \underset{i}{\rightarrow \mathcal{R}_{1}} C^{\prime}\left[s_{i_{1}}, \ldots, s_{i_{m}}\right]=t_{1}
$$

with $i_{k} \in\{1, \ldots, n\}$ for $k=1, \ldots, m$ and $C^{\prime}[, \ldots$,$] is again a context containing$ only symbols from $\mathcal{C} \cup \mathcal{D}_{1} \cup \mathcal{V}$. Since all proper subterms of $t_{0}$ are irreducible we know in particular that all the $s_{i}, 1 \leq i \leq n$ are irreducible. That means $t_{0}$ as well as $t_{1}$ are irreducible w.r.t. $\mathcal{R}_{2}$. Moreover no innermost $\mathcal{R}$-reduction step in $D$ can take place below some $s_{i}$. Hence any innermost $\mathcal{R}$-reduction step starting with $t_{1}$ must again be an innermost $\mathcal{R}_{1}$-step. By induction we can conclude that $D$ is an $\mathcal{R}$-innermost derivation using only $\mathcal{R}_{1}$-steps in the 'top $\mathcal{R}_{1}$-layer' ${ }^{41}$ This implies that we can transform the infinite $\mathcal{R}_{1}^{\mathcal{F}}$-innermost derivation $D$ into an

\footnotetext{
${ }^{40}$ Note that in this case $\mathcal{R}_{2}$ is in particular a constructor system.

${ }^{41}$ Note that $\mathcal{R}_{1}$-steps may create new $\mathcal{R}_{1}$-redexes.
} 
infinite $\mathcal{R}_{1}^{\mathcal{F}_{1}}$-innermost derivation $D^{\prime}$ as follows: For $t_{0}=C\left[s_{1}, \ldots, s_{n}\right]$ as above we obtain $t^{\prime}$ by replacing the maximal 'alien' subterms $s_{i}, 1 \leq i \leq n$, by fresh variables $x_{i}$ such that $x_{i}=x_{j}$ whenever $s_{i}=s_{j}$. By this transformation we obtain from

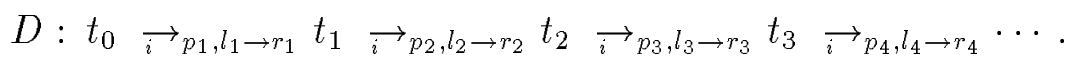

the infinite $\mathcal{R}_{1}^{\mathcal{F}_{1}}$-derivation

$$
D^{\prime}: t_{0}^{\prime} \quad \rightarrow_{p_{1}, l_{1} \rightarrow r_{1}} t_{1}^{\prime} \underset{i}{\longrightarrow} p_{2}, l_{2} \rightarrow r_{2} t_{2}^{\prime} \underset{i}{\rightarrow} p_{3}, l_{3} \rightarrow r_{3} t_{3}^{\prime} \underset{i}{\rightarrow} p_{4}, l_{4} \rightarrow r_{4} \cdots
$$

But this is a contradiction to $I N\left(\mathcal{R}_{1}\right)$.

(c) If $\operatorname{root}\left(t_{0}\right) \in \mathcal{D}_{2}$ we know that $t_{0}$ has the form $t_{0}=C\left[s_{1}, \ldots, s_{n}\right]$ where $C[, \ldots$, is a context containing only symbols from $\mathcal{C} \cup \mathcal{D}_{2} \cup \mathcal{V}$ and $s_{1}, \ldots, s_{n}$ are all the maximal subterms of $t_{0}$ with root symbol in $\mathcal{D}_{1}$. This case is more difficult because the first step in $D$ is an innermost $\mathcal{R}_{2}$-step, i.e. of the form $t_{0} \rightarrow_{i} \mathcal{R}_{2} t_{1}$, which may introduce new innermost $\mathcal{R}_{1}$-redexes due to the possibility of predefined $\mathcal{D}_{1}$-symbols on right-hand sides of $\mathcal{R}_{2}$. We shall show now by induction that $\mathcal{R}_{2}$ is not innermost normalizing which yields a contradiction to the assumption $I N\left(\mathcal{R}_{2}\right)$. To this end we define a measure for the terms $t_{i}$ which is invariant for every innermost $\mathcal{R}_{1}$-step in $D$ and properly decreases for every innermost $\mathcal{R}_{2^{-}}$ step in $D$. The basic idea is to collect the multiset of all maximal subterms $t$ of $t_{i}$ with $\operatorname{root}(t) \in \mathcal{D}_{2}$ which are $\mathcal{R}$-reducible and to compare these multisets by the multiset extension of some appropriate well-founded ordering. Formally we define for every $t_{i}(0 \leq i):^{42}$

$$
M(t):=\left\{\left\{t^{\prime} \mid t^{\prime} \text { is a maximal } \mathcal{R} \text {-reducible subterm of } t \text { with } \operatorname{root}\left(t^{\prime}\right) \in \mathcal{D}_{2}\right\}\right\}
$$

and the ordering

$$
>:=\left(\underset{i}{\rightarrow} \mathcal{R}_{2} \cup>_{s t}\right)^{+},
$$

i.e. $>$ is the transitive closure of the union of the innermost $\mathcal{R}_{2}$-reduction relation and the proper subterm ordering (denoted by $>_{s t}$ ). The (finite) multiset extension of $>$ is denoted by $\gg$ and defined as usual. Since $\vec{i} \mathcal{R}_{2}$ is terminating we know that $>=\left(\underset{i}{\rightarrow} \mathcal{R}_{2} \cup>_{s t}\right)^{+}$is well-founded. Moreover, $\gg$ is well-founded if and only if $>$ is well-founded. Hence, in order to obtain a contradiction it suffices to construct an infinite properly decreasing $\gg$ chain. Consider now some arbitrary step $t_{n} \rightarrow t_{n+1}$ in $D$. We shall prove the following two properties:

$$
\text { (I) } \quad t_{n} \underset{i}{\longrightarrow} \mathcal{R}_{1} t_{n+1} \quad \Longrightarrow M\left(t_{n}\right)=M\left(t_{n+1}\right)
$$

and

$$
\text { (II) } \quad t_{n} \underset{i}{\longrightarrow \mathcal{R}_{2}} t_{n+1} \quad \Longrightarrow \quad M\left(t_{n}\right) \gg M\left(t_{n+1}\right) \text {. }
$$

\footnotetext{
${ }^{42}$ In the following we use double braces in order to distinguish multisets from sets. For the sake of readability the usual set operations are ambiguously used for multisets, too.
} 
From $I N\left(\mathcal{R}_{1}\right)$ we know that only finitely many consecutive steps in $D$ can be $\mathcal{R}_{1}$-innermost steps, hence this implies that $D$ is of the form

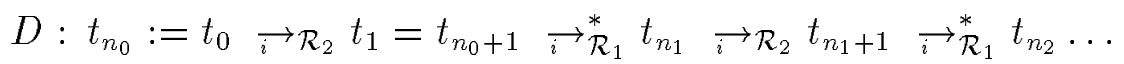

with infinitely many $\underset{i}{\rightarrow} \mathcal{R}_{2}$-steps. But this implies — using (I) and (II) -

$$
M\left(t_{n_{0}}\right) \gg M\left(t_{n_{1}}\right) \gg M\left(t_{n_{2}}\right) \gg \ldots
$$

This is an infinite properly decreasing $\gg$-chain which contradicts $I N\left(\mathcal{R}_{1}\right)$.

It remains to prove the claims (I) and (II) above. (I) follows from the invariant in $D$ that for every $t_{n}$ no $\mathcal{R}_{1}$-rule is applicable below a maximal subterm $t$ of $t_{n}$ with $\operatorname{root}(t) \in \mathcal{D}_{2}$ (this is an easy consequence of the assumption that $\mathcal{R}$ is flat and separated ). (II) is proved by definition of $M($.$) and of >$ as follows. Let $t_{n} \rightarrow_{p, l_{2} \rightarrow r_{2}} t_{n+1}$ with $l_{2} \rightarrow r_{2} \in \mathcal{R}_{2}$. Then we distinguish two cases.

(1) $t_{n} / p \in M\left(t_{n}\right)$, i.e. $t_{n} / p=\sigma\left(l_{2}\right)$ for some substitution $\sigma$ is a maximal reducible subterm of $t_{n}$ with root symbol in $\mathcal{D}_{2}$ : Obviously $t_{n+1} / p=\sigma\left(r_{2}\right)$ is of the form $C\left[s_{1}, \ldots, s_{m}\right]$ with $\operatorname{root}\left(s_{i}\right) \in \mathcal{D}_{2}$ for $1 \leq i \leq m$ and $C[, \ldots$, a context over $\mathcal{C} \cup \mathcal{D}_{1} \cup \mathcal{V}$. By definition of $M$ we get

$$
M\left(t_{n+1}\right) \subseteq\left(M\left(t_{n}\right) \backslash\left\{\left\{t_{n} / p\right\}\right\}\right) \cup\left\{\left\{s_{i} \mid 1 \leq i \leq m\right\}\right\}
$$

Due to $t_{n} / p=\sigma\left(l_{2}\right) \rightarrow_{i} \mathcal{R}_{2} \sigma\left(r_{2}\right)=C\left[s_{1}, \ldots, s_{m}\right]>_{s t} s_{i}$ for all $i, 1 \leq i \leq m$, this implies $M\left(t_{n}\right) \gg M\left(t_{n+1}\right)$.

(2) $t_{n} / p \notin M\left(t_{n}\right)$ : From $t_{n}=\sigma\left(l_{2}\right)$ for some substitution $\sigma$ we know that $t_{n} / p$ is a proper subterm of some maximal $\left(\mathcal{R}_{2}\right.$-reducible) subterm $t_{n} / q$ of $t$ with $\operatorname{root}\left(t_{n} / q\right) \in \mathcal{D}_{2}$. Hence we either have

$$
M\left(t_{n+1}\right)=\left(M\left(t_{n}\right) \backslash\left\{\left\{t_{n} / q\right\}\right\}\right) \cup\left\{\left\{t_{n+1} / q\right\}\right\}
$$

for the case that $t_{n+1} / q$ is $(\mathcal{R}$ - $)$ reducible, or else

$$
M\left(t_{n+1}\right)=M\left(t_{n}\right) \backslash\left\{\left\{t_{n} / q\right\}\right\}
$$

for the case that $t_{n+1} / q$ is $\left(\mathcal{R}_{-}\right)$irreducible.

In both cases we clearly get

$$
M\left(t_{n}\right) \gg M\left(t_{n+1}\right)
$$

Hence the claim is proved which completes the whole proof.

Combining this result with Lemma 4.15 and Theorem 3.20 we obtain as corollary the following invariance result for completeness of hierarchical combinations. 
Theorem 4.25 Let $\mathcal{R}^{\mathcal{F}}=\left(\mathcal{R}_{1} \cup \mathcal{R}_{2}\right)^{\mathcal{F}_{1} \cup \mathcal{F}_{2}}$ be a flat and separated hierarchical combination such that both $\mathcal{R}_{1}^{\mathcal{F}_{1}}$ and $\mathcal{R}_{2}^{\mathcal{F}_{2}}$ are locally confluent overlay systems. Then $\mathcal{R}^{\mathcal{F}}$ is complete if and only if both $\mathcal{R}_{1}^{\mathcal{F}_{1}}$ and $\mathcal{R}_{2}^{\mathcal{F}_{2}}$ are complete.

Theorem 4.25 has already been stated in [Der92] but without proof. A similar result has been presented in [Kri92], too, (cf. Theorem 7, p. 11) but the proof given there for the main technical Lemma (cf. [Kri92] Theorem 6, p.10) is incorrect. One interesting idea presented in [Kri92] is to weaken the 'flatness' condition defined above but retaining 'separation'. The intuition for that is as follows. The crucial point for the 'separation' property defined above is to guarantee that $\mathcal{R}_{2}$-rules do not introduce $\mathcal{D}_{1}$-symbols on right-hand sides of $\mathcal{R}_{2}$-rules below $\mathcal{D}_{2}$-symbols. In order to ensure this property one may weaken the 'flatness' condition by allowing nested $\mathcal{D}_{2}$ symbols on right-hand sides of $\mathcal{R}_{2}$-rules in such a way that (direct or indirect) introduction of $\mathcal{D}_{1}$-symbols below $\mathcal{D}_{2}$-symbols is impossible. This leads us to the following.

Definition 4.26 (cf. [Kri92]) Let $\mathcal{R}^{\mathcal{F}}=\left(\mathcal{R}_{1} \cup \mathcal{R}_{2}\right)^{\mathcal{F}_{1} \cup \mathcal{F}_{2}}$ be the hierarchical combination of the TRSs with constructors $\left(\mathcal{R}_{1}^{\mathcal{F}_{1}}, \mathcal{C}_{1}, \mathcal{D}_{1}\right)$ and $\left(\mathcal{R}_{2}^{\mathcal{F}_{2}}, \mathcal{C}_{2}, \mathcal{D}_{2}\right)$. Then we say that $\mathcal{R}_{2}$ is weakly separated if for every rule $l_{2} \rightarrow r_{2} \in \mathcal{R}_{2}$ and every maximal subterm $t=f\left(t_{1}, \ldots, t_{n}\right)$ of $r_{2}$ with $\operatorname{root}(t)=f \in \mathcal{D}_{2}$ the following holds:

$$
D E P_{\mathcal{R}}(g) \cap \mathcal{D}_{1}=\emptyset \quad \text { for all } g \in\left(\mathcal{D}_{1} \cup \mathcal{D}_{2}\right) \cap \bigcup_{i=1}^{n} F\left(t_{i}\right)
$$

where the set $D E P_{\mathcal{R}}(g)$ of functions symbols on which $g$ 'depends' is defined by $D E P_{\mathcal{R}}(g):=\left\{h \in \mathcal{F} \mid g \rightarrow_{d}^{*} h\right\}$ with $g \rightarrow_{d} h$ if there exists a rule $l \rightarrow r \in \mathcal{R}$ with $\operatorname{root}(l)=g$ and $h \in F(r)$. In this case we also say that the combined system $\mathcal{R}^{\mathcal{F}}$ is weakly separated. ${ }^{43}$

Theorem 4.27 Let $\mathcal{R}^{\mathcal{F}}=\left(\mathcal{R}_{1} \cup \mathcal{R}_{2}\right)^{\mathcal{F}_{1} \cup \mathcal{F}_{2}}$ be a weakly separated hierarchical combination. Then $\mathcal{R}^{\mathcal{F}}=\left(\mathcal{R}_{1} \cup \mathcal{R}_{2}\right)^{\mathcal{F}_{1} \cup \mathcal{F}_{2}}$ is innermost normalizing if and only if both $\mathcal{R}_{1}^{\mathcal{F}_{1}}$ and $\mathcal{R}_{2}^{\mathcal{F}_{2}}$ are innermost normalizing.

Proof: The proof is analogous to the proof of Theorem 4.24.

As a straightforward consequence of Theorem 4.27 above we obtain the following result which is a slight generalization of both Theorem 4.25 (cf. Theorem 24 in [Der92]) and Theorem 7 in [Kri92].

Theorem 4.28 Let $\mathcal{R}^{\mathcal{F}}=\left(\mathcal{R}_{1} \cup \mathcal{R}_{2}\right)^{\mathcal{F}_{1} \cup \mathcal{F}_{2}}$ be a weakly separated hierarchical combination such that both $\mathcal{R}_{1}^{\mathcal{F}_{1}}$ and $\mathcal{R}_{2}^{\mathcal{F}_{2}}$ are locally confluent overlay systems. Then $\mathcal{R}^{\mathcal{F}}$ is complete if and only if both $\mathcal{R}_{1}^{\mathcal{F}_{1}}$ and $\mathcal{R}_{2}^{\mathcal{F}_{2}}$ are complete.

Note that Theorem 4.28 is not in contradiction to Example 4.20. There, applying Theorem 4.27 yields innermost termination of the disjoint union $\mathcal{R}^{\mathcal{F}}=\left(\mathcal{R}_{1} \cup \mathcal{R}_{2}\right)^{\left(\mathcal{F}_{1} \cup \mathcal{F}_{2}\right)}$

\footnotetext{
${ }^{43}$ If $\mathcal{R}_{2}$ is weakly separated and both $\mathcal{R}_{1}$ and $\mathcal{R}_{2}$ are additionally constructor systems then $\mathcal{R}_{2}$ is said to be a proper extension of $\mathcal{R}_{1}$ in [Kri92].
} 
of both TRSs $\mathcal{R}_{1}^{\mathcal{F}_{1}}$ and $\mathcal{R}_{2}^{\mathcal{F}_{2} 44}$. On the other hand we have seen that $\mathcal{R}^{\mathcal{F}}$ is nonterminating. But this is not a contradiction to Theorem 4.28 above since $\mathcal{R}_{1}^{\mathcal{F}_{1}}$ is not an overlay system which means that Theorem 4.28 is not applicable. In other words, innermost termination may be preserved, but for the implication $I N(\mathcal{R}) \Longrightarrow S N(\mathcal{R})$ we need stronger conditions (e.g. $O S(\mathcal{R}) \wedge J C P(\mathcal{R}))$.

\section{Discussion, Related Work and Open Problems}

As noticed by Dershowitz (cf. [Der92]) (one of) the first to consider modularity issues in rewriting was Bidoit ([Bid81]) with his 'gracious' conditions. He proved in [Bid81] that completeness is modular for orthogonal, fully defined ${ }^{45}$ constructor systems. Other interesting results concerning termination of rewriting are investigated by Dershowitz [Der92] and Geupel [Geu89] by means of overlap (and forward) closures. In particular, it is shown in [Geu89] that — as conjectured in [Der81] — a non-overlapping TRS is terminating if and only if no-right-hand side of a forward closure initiates an infinite derivation. This implies as an easy consequence that completeness is modular for non-overlapping TRS (cf. Theorem 4.3 above) as noticed in [Der92]. Moreover, the following generalization of the above result about forward closures is stated in [Der92](cf. Proposition 17): ${ }^{46}$ A uniquely normalizing overlay system is terminating if and only if no right-hand side of a forward closure initiates an infinite derivation. ${ }^{47}$ This result can be used alternatively to provide a proof of our Theorem 4.4 above as sketched in [Der92]. Furthermore some other known and new results about termination of (nondisjoint) combinations of TRSs are summarized in [Der92]. In particular Theorem 4.25 is mentioned there but without proof. As already mentioned a similar result has also been presented in [Kri92], but with an incorrect proof.

Before concluding now let us finally discuss the general idea underlying the approach presented and some open problems. In fact, what has been done in section 3 , is on the one hand side an abstract analysis of the interrelation between restricted and uniform termination under some additional assumptions. On the other hand the goal of the analysis and the results obtained may be considered to be a kind of 'modular' approach to modular properties of TRSs in the following sense. We wanted to find properties $P$ such that e.g.

$$
I N+P \quad \Longrightarrow \quad S N(+P)
$$

holds. The additional knowledge available was that $I N$ is modular. Generalizing this situation we get the following abstract 'modular' approach for obtaining sufficient conditions for the modularity of some property $Q$. Assume that $Q$ is not modular.

\footnotetext{
${ }^{44}$ Note that this follows already from Lemma 4.2 .

${ }^{45}$ This means that every ground term can be reduced to a constructor ground term.

${ }^{46}$ In [Der92] no formal proof for this result is given but some proof idea which roughly spoken says that the proof of [Geu89] can be adapted to the more general case of uniquely normalizing overlay systems.

${ }^{47}$ Here, a TRS is said to be uniquely normalizing if no term has more than one normal form.
} 
Then look for other modular properties $P_{1}, \ldots, P_{n}$ with ${ }^{48}$

$$
\left(P_{1}+\cdots+P_{n}\right) \quad \Longrightarrow \quad Q
$$

Then the property $\left(P_{1}+\cdots+P_{n}+Q\right)$ is obviously modular, hence $Q$ is modular for the class of TRSs satisfying $\left(P_{1}+\cdots+P_{n}\right)$.

Unfortunately, this kind of modular approach is not easily applicable to the main result of [TKB89] which says that $(L L+C O N F+S N)$ is a modular property of TRSs.

For obtaining a modular proof here we would have to find some property $P$ (probably a kind of restricted termination property) satisfying

(1) $(L L+C O N F+P)$ is modular, and

$$
\text { (2) }(L L+C O N F+P) \Longrightarrow S N \text {. }
$$

Since $P$ cannot be $I N$, this is an interesting open problem. But note that even for the case that such a (hopefully easy to understand) property $P$ exists it is not clear a priori that the proofs of (1) and (2) would be simple.

We suppose that it should be possible to extend our approach and proof ideas to the more general case of conditional TRSs. This might in particular also yield a relatively simple proof of the fact that completeness is a decomposable property of conditional constructor systems, a result which has recently been obtained in [Mid93]. Moreover our abstract results also provide a thorough theoretical basis for investigating applications like (termination and uniqueness properties of) different function definition formalisms and inductive theorem proving problems.

\section{Conclusion}

We have provided an abstract analysis of how various kinds of restricted termination (and confluence) properties are related to uniform termination (and confluence). In particular, we have proved some new results about sufficient criteria for termination (strong normalization) which can be considered as generalizations of known results about orthogonal TRSs. Moreover we have shown how these results can be applied in a modular fashion in order to derive simple modular proofs of known and new results concerning modular properties of term rewriting systems. Even more generally we have shown how to obtain invariance results for termination and confluence of certain non-disjoint combinations of TRSs.

Acknowledgements: I would like to thank Klaus Madlener and Claus-Peter Wirth for useful hints and fruitful discussons on early versions of this paper. Moreover I'm grateful to Andrea Sattler-Klein for a thorough reading of a final draft and for detailed criticisms.

\footnotetext{
${ }^{48}$ Slightly weaker, it even suffices that only the conjunction $\left(P_{1}+\cdots+P_{n}\right)$ is modular.
} 


\section{References}

[Bid81] M. Bidoit. Une méthode de présentation de types abstraits: Applications. Thèse de troisième cycle, Université de Paris-Sud, Orsay, France, 1981.

[BKM89] J.A. Bergstra, J.W. Klop, and A. Middeldorp. Termherschrijfsystemen. Technical report, Kluwer Bedrijfswetenschappen, Deventer, 1989. In Dutch.

[Der81] N. Dershowitz. Termination of linear rewriting systems. In S. Even and O. Kariv, editors, Proc. of the 8th Int. Conf. on Automata, Languages and Programming, volume 115 of Lecture Notes in Computer Science, pages 448458. Springer, 1981.

[Der87] N. Dershowitz. Termination of rewriting. Journal of Symbolic Computation, $3(1): 69-116,1987$.

[Der92] N. Dershowitz. Hierarchical termination, Department of Computer Science, Hebrew University, Jerusalem, Israel. Draft version, December 1992.

[DJ90] N. Dershowitz and J.-P. Jouannaud. Rewrite systems. In J. van Leeuwen, editor, Formal models and semantics, Handbook of Theoretical Computer Science, volume B, chapter 6, pages 243-320. Elsevier - The MIT Press, 1990.

[Dro89] K. Drosten. Termersetzungssysteme. Informatik-Fachberichte 210. Springer, 1989. In German.

[Geu89] O. Geupel. Overlap closures and termination of term rewriting systems. Technical Report MIP-8922, Fakultät für Informatik, Universität Passau, July 1989.

[Gra91] B. Gramlich. A structural analysis of modular termination of term rewriting systems. SEKI Report SR-91-15, Dept. of Comp. Science, Univ. of Kaiserslautern, 1991.

[Gra92a] B. Gramlich. Generalized sufficient conditions for modular termination of rewriting. In H. Kirchner and G. Levi, editors, Proc. of 3rd Int. Conf. on Algebraic and Logic Programming, Pisa, Italy, volume 632 of Lecture Notes in Computer Science, pages 53-68. Springer-Verlag, 1992.

[Gra92b] B. Gramlich. Relating innermost, weak, uniform and modular termination of term rewriting systems. In A. Voronkov, editor, International Conference on Logic Programming and Automated Reasoning, St. Petersburg, volume 624 of Lecture Notes in Artificial Intelligence, pages 285-296. Springer-Verlag, 1992.

[Gra92c] B. Gramlich. Sufficient conditions for modular termination of conditional term rewriting systems. In M. Rusinowitch and J.L. Remy, editors, Proc. of the 3rd International Workshop on Conditional Term Rewriting Systems, Pontà-Mousson, volume 656 of Lecture Notes in Computer Science, pages 128-142. Springer-Verlag, 1992. 
[Gra93] B. Gramlich. Generalized sufficient conditions for modular termination of rewriting. Applicable Algebra in Engineering, Communication and Control, 1993. To appear.

[HL78] G. Huet and D. Lankford. On the uniform halting problem for term rewriting systems. Technical Report 283, INRIA, 1978.

[Hue80] G. Huet. Confluent reductions: Abstract properties and applications to term rewriting systems. Journal of the ACM, 27(4):797-821, oct 1980.

[KB70] D.E. Knuth and P.B. Bendix. Simple word problems in universal algebra. In J. Leech, editor, Computational Problems in Abstract Algebra, pages 263-297. Pergamon Press, Oxford, U. K., 1970. Reprinted 1983 in "Automation of Reasoning 2", Springer, Berlin, pp. 342-376.

[KK90] M. Kurihara and I. Kaji. Modular term rewriting systems and the termination. Information Processing Letters, 34:1-4, 1990.

[Klo87] J.W. Klop. Term rewriting systems: A tutorial. Bulletin of the European Association for Theoretical Computer Science, 32:143-182, 1987.

[Klo92] J.W. Klop. Term rewriting systems. In S. Abramsky, D. Gabbay, and T. Maibaum, editors, Handbook of Logic in Computer Science, volume 2, chapter 1, pages 2-117. Clarendon Press, Oxford, 1992.

[KO90a] M. Kurihara and A. Ohuchi. Modularity of simple termination of term rewriting systems. Journal of IPS, Japan, 34:632-642, 1990.

[KO90b] M. Kurihara and A. Ohuchi. Modularity of simple termination of term rewriting systems with shared constructors. Technical Report SF-36, Hokkaido University, Sapporo, 1990. Also in TCS 103, pp. 273-282, 1992.

[Kri92] Krishna Rao, M.R.K. Modular proofs for completeness of hierarchical systems, Computer Science Group, Tata Institute of Fundamental Research, Bombay, India. Draft version, December 1992.

[Mid89] A. Middeldorp. A sufficient condition for the termination of the direct sum of term rewriting systems. In Proceedings of the 4th IEEE Symposium on Logic in Computer Science, pages 396-401, Pacific Grove, 1989.

[Mid90] A. Middeldorp. Modular Properties of Term Rewriting Systems. PhD thesis, Free University, Amsterdam, 1990.

[Mid93] A. Middeldorp. Completeness of combinations of conditional constructor systems. In M. Rusinowitch and J.L. Remy, editors, Proc. of the 3rd International Workshop on Conditional Term Rewriting Systems, Pont-à-Mousson, volume 656 of Lecture Notes in Computer Science, pages 82-96. Springer-Verlag, 1993. 
[MT91] A. Middeldorp and Y. Toyama. Completeness of combinations of constructor systems. In R.V. Book, editor, Proc. of the 4th Int. Conf. on Rewriting Techniques and Applications, volume 488 of Lecture Notes in Computer Science, pages 174-187. Springer, 1991.

[O'D77] M.J. O'Donnell. Computing in Systems Described by Equations, volume 58 of Lecture Notes in Computer Science. Springer, 1977.

[O'D85] M.J. O'Donnell. Equational logic as a programming language. MIT Press, 1985 .

[Oh193] E. Ohlebusch. On the modularity of termination of term rewriting systems. Technical Report 11, Universität Bielefeld, March 1993.

[Ros73] B.K. Rosen. Tree-manipulating systems and Church-Rosser theorems. Journal of the ACM, 20:160-187, 1973.

[Rus87] M. Rusinowitch. On termination of the direct sum of term rewriting systems. Information Processing Letters, 26:65-70, 1987.

[TKB89] Y. Toyama, J.W. Klop, and H.P. Barendregt. Termination for the direct sum of left-linear term rewriting systems. In N. Dershowitz, editor, Proc. of the 3rd Int. Conf. on Rewriting Techniques and Applications, volume 355 of Lecture Notes in Computer Science, pages 477-491. Springer, 1989.

[Toy87a] Y. Toyama. Counterexamples to termination for the direct sum of term rewriting systems. Information Processing Letters, 25:141-143, 1987.

[Toy87b] Y. Toyama. On the Church-Rosser property for the direct sum of term rewriting systems. Journal of the ACM, 34(1):128-143, 1987. 